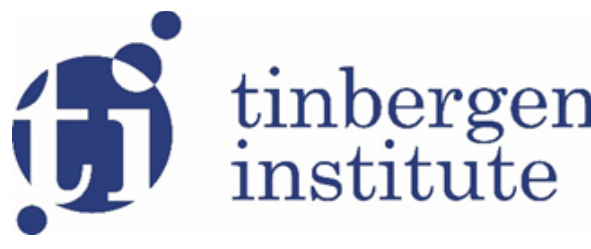

TI 2019-076/II

Tinbergen Institute Discussion Paper

\section{Sequential bankruptcy problems}

\author{
Arantza Estévez-Fernández ${ }^{1}$ \\ José Manuel Giménez-Gómez ${ }^{2}$ \\ María José Solís-Baltadano ${ }^{2}$
}

1 Tinbergen Institute and Department of Econometrics and Operations Research, VU Amsterdam, The Netherlands

2 Department of Economics, Universitat Rovira i Virgili, Spain 
Tinbergen Institute is the graduate school and research institute in economics of Erasmus University Rotterdam, the University of Amsterdam and VU University Amsterdam.

Contact: discussionpapers@tinbergen.nl

More TI discussion papers can be downloaded at https://www.tinbergen.nl

Tinbergen Institute has two locations:

Tinbergen Institute Amsterdam

Gustav Mahlerplein 117

1082 MS Amsterdam

The Netherlands

Tel.: +31(0)205984580

Tinbergen Institute Rotterdam

Burg. Oudlaan 50

3062 PA Rotterdam

The Netherlands

Tel.: +31(0)10408 8900 


\title{
Sequential bankruptcy problems
}

\author{
A. Estévez-Fernández ${ }^{1} \quad$ J.M. Giménez Gómez ${ }^{2} \quad$ M.J. Solís Baltodano \\ ${ }^{1}$ Tinbergen Institute and Department of Econometrics and Operations Research, VU Amsterdam, The Netherlands. \\ 2 Department of Economics, Universitat Rovira i Virgili, Spain.
}

\begin{abstract}
In this paper, we analyze sequential bankruptcy problems, which generalize bankruptcy problems. They contain the problems of sharing water in a transboundary river and of allocating expedition rewards in projects. We provide three mechanisms for generalizing rules for bankruptcy problems to rules for sequential bankruptcy problems: the upwards, the downwards, and the two-steps mechanisms. Further, we characterize the upwards constrained equal awards, the upwards constrained equal losses, and the upwards proportional rules on the basis of upwards composition and upwards path independence. Moreover, we compare the three mechanisms based on inheritance of well-established properties for bankruptcy rules to the setting of sequential bankruptcy rules.
\end{abstract}

Keywords: Sequential bankruptcy, upwards mechanism, downwards mechanism, twosteps mechanism, constrained equal awards rule, constrained equal losses rule, proportional rule

\section{Introduction}

Bankruptcy problems are one of the simplest, yet most interesting, economic problems. In a bankruptcy problem, there is a group of agents with rightful claims over a scarce estate. The question is how to "fairly" share the estate among the agents. There is an extensive literature on bankruptcy problems from a game theoretical perspective (cf. O'Neill 1982, Aumann and Maschler 1985, Curiel et al. 1987, as seminal papers) as well as from an axiomatic perspective (cf. Moulin 1987, Dagan 1996, Moulin 2000, Herrero and Villar 2001). For a survey on bankruptcy literature we refer to Thomson $(2003 ; 2015)$.

Bankruptcy problems have been applied in a variety of economic situations like in sharing the emission of $\mathrm{CO}_{2}$ (Duro Moreno et al. 2018), passepartout problems (Estévez-Fernández et al. 2012), museum pass problems (Casas-Mendez et al. 2011; 2014, Bergantiños and MorenoTernero 2015; 2016) and in two-sided matching problems (Estévez-Fernández et al. 2016). Recently, Ansink and Weikard (2012) analyses the problem of sharing water in a transboundary river in the framework of bankruptcy problems where the agents are linearly ordered. This linear order is given by the position in the river and the natural flow of its water. Moreover, 
at each location along the river, there is exactly one agent and the total water inflow at each location restricts the maximum amount of water an agent can get. Allowing more than one agent at each location enriches the problem since allows the representation of its different uses, e.g. human consumption, crop irrigation, electricity, industry, etc. By allowing to differentiate amongst water uses, it is possible to take priorities of claimants during drought times (Moulin 2000, Thomson 2003).

Estévez-Fernández et al. (2007), and Estévez-Fernández (2012) in a more general setting, analyze allocation of delay costs and expedition rewards in projects within a game theoretical framework. In many projects, specially when developing high technology, there is a race against the clock and high incentives are given in order to finish the project before the planned time. For a project to be expedited, the activities need to coordinate and cooperate for this aim. Estévez-Fernández et al. (2007) and Estévez-Fernández (2012) model the allocation of revenues from expedited projects using a bankruptcy approach where the agents have a linear order based on the minimum slack of all the "paths" in which they are involved. Besides, the slack of the paths also restrict the maximum amount the activities in a path can obtain from the total reward.

In this paper, we introduce sequential bankruptcy problems, which form a theoretical framework that supports the works of Ansink and Weikard (2012), Estévez-Fernández et al. (2007), and Estévez-Fernández (2012). In a sequential bankruptcy problem, there is an ordered partition of the set of agents, $\left\langle N_{1}, \ldots, N_{r}\right\rangle$, and $r$ estates, $E_{1}, \ldots, E_{r}$, such that the members in $N_{1}$ claim on $E_{1}$, the members in $N_{2}$ claim on $E_{1}$ and $E_{2}$, and so forth. Moreover, each estate $E_{l}$ is not high enough to satisfy the total claim of the members in $N_{l}, \ldots, N_{r}$. Relating it back to the work of Ansink and Weikard (2012), the problem of sharing water in a transboundary river can be modeled as a sequential bankruptcy problem where $\left|N_{l}\right|=1$ for each $l \in\{1, \ldots, r\}$. Regarding the work of Estévez-Fernández (2012), the allocation of revenues from expedited projects can be modeled as a sequential bankruptcy problem where: (i) there are as many elements in the partition of agents as groups of paths with same slack that are involved in the project expedition and (ii) the total estate over which a group can claim is the contribution of the group to the total reward of the expedition.

We introduce three mechanisms for generalizing rules for bankruptcy problems to rules for sequential bankruptcy problems: the upwards, the downwards, and the two-steps mechanisms. These names are inspired in the literature of sharing water in a river. Given a bankruptcy rule $f$, the upwards mechanism generates a sequential bankruptcy rule by starting to share the estate "downstream" and, subsequently, moving upwards while updating the claims at each stage of the procedure. The downwards mechanism generalizes the mechanism in Ansink and Weikard (2012). Given a bankruptcy rule $f$, the downwards mechanism generates a sequential bankruptcy rule by starting to share the estate "upstream" while considering the total claim of the "downstream" groups and, subsequently, moving downwards while updating the downwards estate at each stage of the procedure. Finally, the two-steps mechanism is related to the two-step procedure for bankruptcy problems with a priori unions in Borm et al. 
(2005). Given a bankruptcy rule $f$, the two-steps mechanism generates a sequential bankruptcy rule by, in a "first step" applying the rule to the bankruptcy problem with $r$ agents where the estate is the sum of all estates and the claim of agent $l$ is the total claim of the members of $N_{l}$ truncated with respect to the sum of the estates $E_{l}, \ldots, E_{r}$; and in a "second step", applying the rule to divide the allocation of each group $N_{1}, \ldots, N_{r}$ among their members.

Next to describing the three mechanisms above, we focus on the upwards mechanism, which is the most novel one. We focus on three of the most used rules for bankruptcy problems: the proportional, the constrained equal awards, and the constrained equal losses rules. Inspired in Dagan (1996) and Herrero and Villar (2001), we characterize the upwards constrained equal awards rule by means of upwards composition, the upwards constrained equal losses rule by means of upwards path independency, and the upwards proportional rule is characterize using upwards self-duality and upwards composition, as well as upwards self-duality and upwards path independency.

To conclude, we focus on differences among the three mechanisms based on inheritance of well-established properties for bankruptcy rules to the setting of sequential bankruptcy rules. In particular, we see that equal treatment of equals, composition, and path independence are not inherited in the class of sequential bankruptcy problems by the downwards and two-steps mechanisms according to our generalizations. Besides, we show that invariance under claims truncation and minimal rights first are inherited in the class of sequential bankruptcy problems by the downwards and the two-steps mechanisms according to our generalizations.

The remainder of the paper is organized as follows. Section 2 surveys bankruptcy problems and introduces sequential bankruptcy problems. In Section 3, we introduce the upwards mechanism and provide characterizations for the upwards constrained equal awards, the upwards constrained equal losses, and the upwards proportional rules. To conclude, in Section 4, we introduce the downwards and the two-steps mechanisms.

\section{Preliminaries}

In this section, we give a brief survey of existing concepts in the literature of bankruptcy problems and introduce sequential bankruptcy problems.

First, we present notation that will be used throughout the article. For $x \in \mathbb{R}, x_{+}=$ $\max \{0, x\}$. Let $N$ be a finite set. We denote by $0 \in \mathbb{R}^{N}$ the vector with all zeros. For $x \in \mathbb{R}^{N}$ and $S \subseteq N, x_{S} \in \mathbb{R}^{S}$ denotes the projection of $x$ in $\mathbb{R}^{S}$ and $x(S)=\sum_{i \in S} x_{i}$.

In a bankruptcy problem, a finite group of agents have a rightful claim over a scarce estate. Formally, a bankruptcy problem is described by a tuple $(N, E, c)$ where $N$ is the set of agents, $E \in \mathbb{R}_{+}$is the estate, and $c \in \mathbb{R}_{+}^{N}$ is the vector of claims, with $c_{i}$ the claim of $i \in N$ on $E$, that satisfies $c(N) \geq E$. Let $\mathcal{B}^{N}$ denote the set of bankruptcy problems with set of agents $N$. For notational easiness, a bankruptcy problem is denoted $(E, c) \in \mathcal{B}^{N}$.

For $(E, c) \in \mathcal{B}^{N}$, the aggregate loss of a bankruptcy problem is the difference between the total claim and the estate, that is, $L(E, c)=c(N)-E$. If no confusion is to be expected, we 
write $L$ instead of $L(E, c)$. For a claimant $i \in N$, the minimal right of $i$ is the amount of estate available, if any, once all other claimants have received their full claim. Another interpretation of the minimal right of $i$ is that player $i$ is going to pay the aggregate loss. The minimal right of $i$ is, then, the part of the claim that is left, if any, after subtracting the aggregate loss:

$$
m_{i}(E, c)=(E-c(N \backslash\{i\}))_{+}=\left(c_{i}-L\right)_{+} .
$$

If no confusion is to be expected, we write $m_{i}$ instead of $m_{i}(E, c)$. It is well established that $\sum_{i \in N} m_{i} \leq E$ (cf. O’Neill 1982, Curiel et al. 1987).

A bankruptcy rule is a function $f$ that assigns to each bankruptcy problem $(E, c) \in \mathbb{B}^{N}$ a vector $f(E, c) \in \mathbb{R}^{N}$ satisfying

$$
0 \leq f(E, c) \leq c \text { and } \sum_{i \in N} f_{i}(E, c)=E
$$

The three more relevant bankruptcy rules in the literature are the proportional rule, the constrained equal awards rule, and the constrained equal losses rule. The proportional rule, Prop, allocates the estate among the agents proportionally to their claims: For every $(E, c) \in \mathcal{B}^{N}$, $\operatorname{Prop}(E, c)=\frac{E}{c(N)} c$. The constrained equal awards rule, CEA, allocates the estate as equal as possible among the agents, considering that they do not get more than their claims: For every $(E, c) \in \mathcal{B}^{N}, \operatorname{CEA}(E, c)=\left(\min \left\{c_{i}, \alpha\right\}\right)_{i \in N}$ with $\alpha \in \mathbb{R}$ such that $\sum_{i \in N} \min \left\{c_{i}, \alpha\right\}=E$. The constrained equal losses rule, CEL, allocates the losses as equal as possible among the agents, considering that they do not get a negative amount: For every $(E, c) \in \mathcal{B}^{N}, \operatorname{CEL}(E, c)=$ $\left(\left(c_{i}-\beta\right)_{+}\right)_{i \in N}$ with $\beta \in \mathbb{R}$ such that $\sum_{i \in N}\left(c_{i}-\beta\right)_{+}=E$. For a survey on more bankruptcy rules and their properties, we refer to Thomson (2003; 2015).

Before defining sequential bankruptcy problems, we give two introductory examples. Ambec and Sprumont (2002) initiated a mainstream of literature on sharing the water of an international river among the agents located along the river. Ansink and Weikard (2012) further analyses this problem in the framework of bankruptcy problems where the agents are linearly ordered. They propose a mechanism that transforms bankruptcy rules into rules for sharing water in a river. One feature of the problem in Ansink and Weikard (2012) is that in each river location, there is exactly one agent. We now consider that at each location, there may be more than one agent. For instance, in one specific location, one agent might represent the water needed for human consumption, another might represent the water needed for crop irrigation, and another one might represent the water needed for industry. By allowing more than one agent at each river location, we can also take priorities of claimants into account: under water shortage, human consumption may have priority over water needed for industry. For more on bankruptcy problems with priorities, see Moulin (2000) and Thomson (2003).

Example 2.1. Consider a river with three locations, 1,2,3, along the river. We may assume that location 1 is the location that is most upstream, location 2 is the location that is halfway, and location 3 is the 
most downstream one. In location 1 there is a total inflow of $E_{1}=6$ units and there are two claimants, $A$ and $B$, with claims $c_{A}=2$ and $c_{B}=3$; in location 2 there is a total inflow of $E_{2}=5$ units and there are three claimants, $C, D$, and $E$, with claims $c_{C}=1, c_{D}=2$, and $c_{E}=3$; and in location 3 there is a total inflow of $E_{3}=3$ units and there is one claimant, $F$, with claim $c_{F}=5$.

Here, the agents at location 1 have rights over the inflow of 6 units; the agents at location 2 have rights over the total inflow at locations 1 and 2 of $6+5=11$ units; and the agent at location 3 has rights over the total inflow of the river: $6+5+3=14$ units.

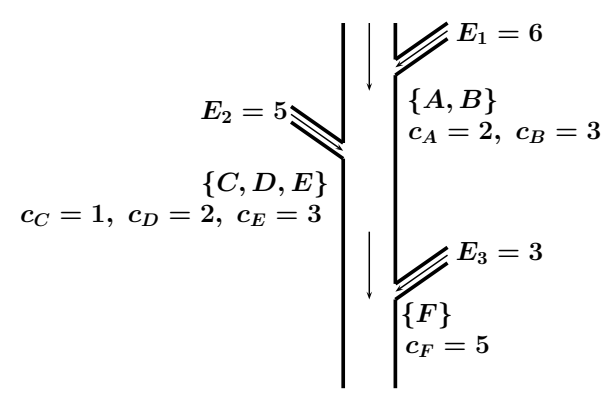

Figure 1: Sharing the water in a river in Example 2.1.

In this specific situation, agents $A$ and $B$ have an inflow that totally satisfies their claims and let 1 unit of water go downstream. The agents $C, D$, and $E$ have a total of $5+1$ units of water inflow, in order to satisfy their demands. Agent $F$ has a claim of 5 and an insufficient inflow of 3 units to satisfy his claim. Therefore, agents $A, B, C, D$ and $E$ may not be able to fulfill all their claims.

Estévez-Fernández et al. (2007), and Estévez-Fernández (2012) in a more general setting, analyze allocation of delay costs and expedition rewards in projects within a game theoretical framework. A project consists of a set of activities that are interconnected and need to be carried out during a period of time in order to achieve a particular aim. Examples of projects are the construction of a building, the organization of a congress, or the development of the hyperloop. A planned project specifies its activities, their interconnections, and the planned time to carry out each activity. This allows us to have a planned duration of the project. In many projects, specially when developing high technology, there is a race against the clock and high incentives are given in order to finish the project before the planned time. For a project to be expedited, the activities need to coordinate and cooperate for this aim as the following example illustrates.

Example 2.2. Consider a project with six activities which interconnections are given in Figure 2.

\begin{tabular}{|l|l|}
\hline Activity & Predecessors \\
\hline A & \\
\hline B & A \\
\hline C & \\
\hline D & \\
\hline E & D \\
\hline F & D, E \\
\hline
\end{tabular}

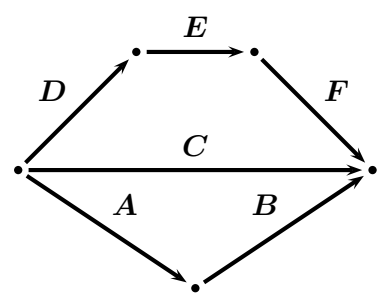

Figure 2: Activities interconnections and project representation in Example 2.2. 
The planned time of the activities are $p(A)=5, p(B)=7, p(C)=10, p(D)=4, p(E)=2$ and $p(F)=3$. Hence, the planned time of the project is

$$
\max \{p(A)+p(B), p(C), p(D)+p(E)+p(F)\}=\max \{5+7,10,4+2+3\}=12
$$

The manager wants the project to be expedited. For this, he is willing to pay a reward of $€ 1000$ per unit of expedition. To expedite the whole project, activities in path $A-B$ need to be expedited. If only activities in $A-B$ are expedited and $C, D, E, F$ act according to plan, the project can have a maximum expedition of 2 units of time. If $C$ coordinates with $A$ and $B$ to expedite the project, while $D, E$, and $F$ act according to plan, they could increase the total expedition by at most one extra unit of time. To further expedite the project, $A, B$ and $C$ also need the cooperation of activities in the path D-E-F. Let the realization times of the project be those given on Table 1.

\begin{tabular}{|c|c|c|c|}
\hline Activity & $\begin{array}{c}\text { Planned } \\
\text { time }\end{array}$ & $\begin{array}{c}\text { Realization } \\
\text { time }\end{array}$ & Expedition \\
\hline A & 5 & 2 & 3 \\
\hline B & 7 & 3 & 4 \\
\hline C & 10 & 7 & 3 \\
\hline D & 4 & 3 & 1 \\
\hline E & 2 & 1 & 1 \\
\hline F & 3 & 2 & 1 \\
\hline \hline Path & & & \\
\hline A-B & $\mathbf{1 2}$ & 5 & \\
\hline C & 10 & $\mathbf{7}$ & \\
\hline D-E-F & 9 & 6 & \\
\hline
\end{tabular}

Table 1: Planned and realization times of the activities and the paths in Example 2.2.

In this situation, A claims $€ 3000$, B claims $€ 4000$, C claims $€ 3000$, and D, E, and F claim $€ 1000$ each. Here, $A$ and $B$ are needed to bring the total expedition of the project $(12-7=5)$, and have rights over the total reward of $€ 5000$. Activity $C$ contributes to the expedition of $10-7=3$ units of time and has rights over $€ 3000$ of the total reward. Activities $D, E$, and F contribute to the expedition of $9-7=2$ units of time and have rights over $€ 2000$ of the total reward.

We now introduce sequential bankruptcy problems. Let $N$ be a finite set of claimants and let $\left\langle N_{1}, \ldots, N_{r}\right\rangle$ be an ordered partition of $N$. A sequential bankruptcy problem is a tuple $\left(\left\langle N_{1}, \ldots, N_{r}\right\rangle,\left(E_{1}, \ldots, E_{r}\right), c\right)$ where $E_{1}, \ldots, E_{r} \in \mathbb{R}_{+}$are the estates to be shared among the claimants such that the members of $N_{1}$ claim over $E_{1}$, the members of $N_{2}$ claim over $E_{1}+E_{2}$, and, in general, the members of $N_{l}$ claim over $\sum_{\lambda=1}^{l} E_{\lambda}$ for $l \in\{1, \ldots, r\}$; and $c \in \mathbb{R}_{+}^{N}$ is the vector of claims satisfying $c(N) \geq \sum_{\lambda=1}^{r} E_{\lambda}$. Similar to Ansink and Weikard (2012), we impose the following assumption to guarantee meaningfulness of sequential bankruptcy problems. 


\section{Assumption:}

$$
\sum_{\lambda=l}^{r} c\left(N_{\lambda}\right) \geq \sum_{\lambda=l}^{r} E_{\lambda} \quad \text { for all } l \in\{1, \ldots, r\}
$$

Let $\mathcal{B}^{N_{1}, \ldots, N_{r}}$ denote the set of bankruptcy problems with set of agents $N=\bigcup_{\lambda=1}^{r} N_{\lambda}$ and ordered partition $\left\langle N_{1}, \ldots, N_{r}\right\rangle$. For notational easiness, a sequential bankruptcy problem is denoted $(\underline{E}, c) \in \mathcal{B}^{N_{1}, \ldots, N_{r}}$, where $\underline{E}=\left(E_{1}, \ldots, E_{r}\right)$. Moreover, for $k \in\{1, \ldots, r\}$, let

$$
\underline{E_{k}}=\left(0, \ldots, 0, E_{k}, 0, \ldots, 0\right) \text { and } \underline{E}^{k}=\left(0, \ldots, 0, E_{k}, \ldots, E_{r}\right) \text {. }
$$

For $(\underline{E}, c) \in \mathcal{B}^{N_{1}, \ldots, N_{r}}$, the aggregate loss for the members of $N_{k}$ is defined by $L_{k}(\underline{E}, c)=$ $c\left(N_{k}\right)-E_{k}$. Unlike in bankruptcy problems, $L_{k}(\underline{E}, c)$ may be negative. If no confusion is to be expected, we write $L_{k}$ instead of $L_{k}(\underline{E}, c)$. Our assumption can now be restated as

$$
\sum_{\lambda=l}^{r} L_{\lambda} \geq 0 \text { for all } l \in\{1, \ldots, r\} \text {. }
$$

Example 2.3. The problem of sharing water in a river in Example 2.1 can be seen as a sequential bankruptcy problem where $N=\{A, B, C, D, E, F\}, N_{1}=\{A, B\}, N_{2}=\{C, D, E\}$, and $N_{3}=\{F\}$; $E_{1}=6, E_{2}=5$, and $E_{3}=3$; and $c=(2,3,1,2,3,5)$.

The expedition project problem in Example 2.2 can be seen as a sequential bankruptcy problem where $N=\{A, B, C, D, E, F\}, N_{1}=\{D, E, F\}, N_{2}=\{C\}$, and $N_{3}=\{A, B\} ; E_{1}=2000, E_{2}=1000$, and $E_{3}=2000 ;$ and $c=(3000,4000,3000,1000,1000,1000)$.

A sequential bankruptcy rule or rule is a function $f$ that assigns to each sequential bankruptcy problem $(\underline{E}, c) \in \mathcal{B}^{N_{1}, \ldots, N_{r}}$ a vector $f(\underline{E}, c) \in \mathbb{R}^{N}$ satisfying

$$
\begin{aligned}
& 0 \leq f(\underline{E}, c) \leq c \\
& \sum_{\lambda=1}^{l} \sum_{i \in N_{\lambda}} f_{i}(\underline{E}, c) \leq \sum_{\lambda=1}^{l} E_{\lambda} \text { for each } l=1, \ldots, r-1, \text { and } \\
& \sum_{\lambda=1}^{r} \sum_{i \in N_{\lambda}} f_{i}(\underline{E}, c)=\sum_{\lambda=1}^{r} E_{\lambda} .
\end{aligned}
$$

For $k \in\{1, \ldots, r\}$ and $E_{1}=\ldots=E_{k}=0$, conditions (1) and (2) imply $x_{N_{l}}=\mathbf{0}_{N_{l}}$ for each $l \in\{1, \ldots, k\}$.

One way to discern among several division rules is by looking to their properties. We now pay attention to generalizing basic properties for bankruptcy rules to properties for sequential bankruptcy rules. We first consider invariance under claims truncation (see Curiel, Maschler and Tijs 1987). The question we first need to address is what is our reference point for truncation. It is clear that claimants can never get more than the "total estate" over which they are claiming. 
A rule $f$ satisfies invariance under claims truncation if for each $(\underline{E}, c) \in \mathcal{B}^{N_{1}, \ldots, N_{r}}$,

$$
f(\underline{E}, c)=f\left(\underline{E}, c^{\underline{E}}\right)
$$

where $c_{i}^{E}=\min \left\{c_{i}, \sum_{\lambda=1}^{k} E_{\lambda}\right\}$ for all $i \in N_{k}$ and all $k \in\{1, \ldots, r\}$.

A rule $f$ satisfies weak invariance under claims truncation if for each $k \in\{1, \ldots, r\}$ and each $\left(\underline{E_{k}}, c\right) \in \mathcal{B}^{N_{1}, \ldots, N_{r}}$,

$$
f\left(\underline{E_{k}}, c\right)=f\left(\underline{E_{k}}, c^{E_{k}}\right)
$$

where $c_{i}^{E_{k}}=\min \left\{c_{i}, E_{k}\right\}$ for all $i \in \bigcup_{\lambda=k}^{r} N_{\lambda}$ and $c_{i}^{E_{k}}=0$ for all $i \in \bigcup_{\lambda=1}^{k-1} N_{\lambda}$. It readily follows that invariance under claims truncation implies weak invariance under claims truncation.

Next, we consider equal treatment of equals (cf. Thomson 2003). First, we need to decide when two claimants are considered equal. For a start, they need to have equal claims. Besides, they also need to hold their claim over the same amount of "total estate". A rule $f$ satisfies equal treatment of equals if for each $(\underline{E}, c) \in \mathcal{B}^{N_{1}, \ldots, N_{r}}$, each $k, l \in\{1, \ldots, r\}$ with $\sum_{\lambda=1}^{k} E_{\lambda}=$ $\sum_{\lambda=1}^{l} E_{\lambda}$, and each $i \in N_{k}$ and $j \in N_{l}$ with $c_{i}=c_{j}$,

$$
f_{i}(\underline{E}, c)=f_{j}(\underline{E}, c)
$$

Therefore, if $i \in N_{k}$ and $j \in N_{l}$, with $k<l$, are equals, then, $c_{i}=c_{j}$ and $E_{k+1}=\ldots=E_{l}=0$.

To conclude, we generalize minimal rights first (cf. Thomson 2003). The minimal right of claimant $i \in N_{k}$ is the part of the estate left, if any, after all other claimants have been fully compensated. For this, we first need to define the additional estate that is available to $N_{k}$ from the upstream claimants. Let $(\underline{E}, c) \in \mathcal{B}^{N_{1}, \ldots, N_{r}}$ and let $A E_{0}=0$. For $l=1$,

$$
A E_{1}=\left(A E_{0}+E_{1}-c\left(N_{1}\right)\right)_{+}=\left(A E_{0}-L_{1}\right)_{+}
$$

is the part of the estate $E_{1}$ that the members of $N_{1}$ are not claiming and is the additional estate for $N_{2}$. Then, the members of $N_{2}$ can guarantee a claim over $E_{2}+A E_{1}$. For $l \in\{2, \ldots, r-1\}$,

$$
A E_{l}=\left(A E_{l-1}+E_{l}-c\left(N_{l}\right)\right)_{+}=\left(A E_{l-1}-L_{l}\right)_{+}
$$

is the additional estate for $N_{l+1}$ and the members of $N_{l+1}$ can guarantee a claim over $E_{l+1}+A E_{l}$. By the assumption on sequential bankruptcy problems, $A E_{l}-\sum_{\lambda=l+1}^{r} L_{\lambda} \leq 0$ for $l \in\{1, \ldots, r-1\}$.

The minimal right of $i \in N_{k}, k \in\{1, \ldots, r\}$, is defined by

$$
\begin{aligned}
m_{i}(\underline{E}, c) & =\left(A E_{k-1}+E_{k}-c\left(N_{k} \backslash\{i\}\right)+\sum_{\lambda=k+1}^{r}\left(E_{\lambda}-c\left(N_{\lambda}\right)\right)\right)_{+} \\
& =\left(A E_{k-1}+c_{i}-\sum_{\lambda=k}^{r} L_{\lambda}\right)_{+}
\end{aligned}
$$


We denote $m(\underline{E}, c)=\left(m_{i}(\underline{E}, c)\right)_{i \in N}$. If no confusion is to be expected, we write $m_{i}$ instead of $m_{i}(\underline{E}, c)$ and $m$ instead of $m(\underline{E}, c)$.

A rule $f$ satisfies minimal rights first if for each $(\underline{E}, c) \in \mathcal{B}^{N_{1}, \ldots, N_{r}}$,

$$
f(\underline{E}, c)=m+f\left(\bar{E}_{1}-\sum_{i \in N_{1}} m_{i}, \ldots, \bar{E}_{r}-\sum_{i \in N_{r}} m_{i}, c-m\right)
$$

with $\bar{E}_{l}=\min \left\{c\left(N_{l}\right), A E_{l-1}+E_{l}\right\}$ for $l \in\{1, \ldots, r\}$.

Easily, $m\left(\bar{E}_{1}, \ldots, \bar{E}_{r}, c\right)=m(\underline{E}, c)$. Therefore, minimal rights first is very restrictive since it implies $f(\underline{E}, c)=f\left(\bar{E}_{1}, \ldots, \bar{E}_{r}, c\right)$ for every $(\underline{E}, c) \in \mathcal{B}^{N_{1}, \ldots, N_{r}}$. We weaken the property as follows. A rule $f$ satisfies weak minimal rights first if for each $k \in\{1, \ldots, r\}$ and each $\left(\underline{E_{k}}, c\right) \in$ $\mathcal{B}^{N_{1}, \ldots, N_{r}}$,

$$
f\left(\underline{E_{k}}, c\right)=m+f\left(0, \ldots, 0, E_{k}-\sum_{\lambda=k}^{r} \sum_{i \in N_{\lambda}} m_{i}, 0, \ldots, 0, c-m\right) .
$$

Readily, minimal rights first implies weak minimal rights first.

The following result provides an expression of the minimal right of the claimants based on their claim and the aggregate loss on the different sets of claimants. The proof follows straightforwardly from the definition of minimal right and is, therefore, omitted.

Proposition 2.4. Let $(\underline{E}, c) \in \mathcal{B}^{N_{1}, \ldots, N_{r}}$.

(i) $A E_{k-1}-\sum_{\mu=k}^{r} L_{\lambda}=\max _{1 \leq \lambda \leq k}\left\{-\sum_{\mu=\lambda}^{r} L_{\mu}\right\} \leq 0$ for every $k \in\{2, \ldots, r\}$.

(ii) For $i \in N_{k}, m_{i}(\underline{E}, c)=\max _{1 \leq \lambda \leq k}\left\{\left(c_{i}-\sum_{\mu=\lambda}^{r} L_{\mu}\right)_{+}\right\}$.

\section{Upwards mechanism for sequential bankruptcy problems}

In this section, we introduce the upwards mechanism for sequential bankruptcy problems. It generalizes rules for bankruptcy problems to rules for sequential bankruptcy problems. The name of upwards mechanism is inspired by the connection of sequential bankruptcy problems and problems of sharing water in a river: The mechanism starts allocating the estates from downstream and moves upwards by updating the claims at each stage. We start this section by reconsidering Example 2.1.

Example 3.1. Reconsider the problem of sharing water in a river in Example 2.1 (see Figure 1). As pointed out in Example 2.3, it can be interpreted as a sequential bankruptcy problem with $N=\{A, B, C, D, E, F\}, N_{1}=\{A, B\}, N_{2}=\{C, D, E\}$, and $N_{3}=\{F\} ; E_{1}=6, E_{2}=5$, and $E_{3}=3$; and $c=(2,3,1,2,3,5)$.

To allocate the available water among the claimants, we will use the constrained equal awards rule. First, recall that the water inflow $E_{3}$ can only be shared among the claimants in $N_{3}$ as water only flows 
downstream. We can apply the constrained equal awards rule to $\left(E_{3},\left(\mathbf{0}_{N_{1}}, \mathbf{0}_{N_{2}}, c_{N_{3}}\right)\right) \in \mathcal{B}^{N}$ :

$$
x^{3}=C E A(3,(0,0,0,0,0,5))=(0,0,0,0,0,3) .
$$

Second, the water inflow $E_{2}$ can only be shared among the claimants in $\mathrm{N}_{2}$ and $\mathrm{N}_{3}$ as water only flows downstream. Since in our first step agent $F$ has already got 3 units of water, we need to update his claim to $5-3=2$. We can now apply the constrained equal awards rule to $(5,(0,0,1,2,3,2)) \in \mathcal{B}^{N}$ :

$$
x^{2}=C E A(5,(0,0,1,2,3,2))=\left(0,0,1,1 \frac{1}{3}, 1 \frac{1}{3}, 1 \frac{1}{3}\right) .
$$

Third and last, the water inflow $E_{1}$ can be shared among all the claimants. Since in our second step agents $C, D, E$ and $F$ have already got water, we need to update their claims: for $C$ we have $1-1=0$, for $D$ we have $2-1 \frac{1}{3}=\frac{2}{3}$, for $E$ we have $3-1 \frac{1}{3}=1 \frac{2}{3}$, and for $F$ we have $2-1 \frac{1}{3}=\frac{2}{3}$. We can now apply the constrained equal awards rule to $\left(6,\left(2,3,0, \frac{2}{3}, 1 \frac{2}{3}, \frac{2}{3}\right)\right) \in \mathcal{B}^{N}$ :

$$
x^{1}=\operatorname{CEA}\left(6,\left(2,3,0, \frac{2}{3}, 1 \frac{2}{3}, \frac{2}{3}\right)\right)=\left(1 \frac{5}{9}, 1 \frac{5}{9}, 0, \frac{2}{3}, 1 \frac{5}{9}, \frac{2}{3}\right) \text {. }
$$

The upwards mechanism applied to the constrained equal awards rule leads to the allocation:

$$
x^{3}+x^{2}+x^{1}=\left(1 \frac{5}{9}, 1 \frac{5}{9}, 1,2,2 \frac{8}{9}, 5\right)
$$

To formally describe the upwards mechanism, we need to introduce new notation. Let $(\underline{E}, c) \in \mathcal{B}^{N_{1}, \ldots, N_{r}}$. For $l \in\{1, \ldots, r\}$, let

$$
c^{l}=\left(\mathbf{0}_{N_{1}}, \ldots, \mathbf{0}_{N_{l-1}}, c_{N_{l}}, \ldots, c_{N_{r}}\right) .
$$

Given a bankruptcy rule $f$, the upwards mechanism generates a rule for sequential bankruptcy problems, $f^{\mathrm{Up}}$, that assigns to each $(\underline{E}, c) \in \mathcal{B}^{N_{1}, \ldots, N_{r}}$, a vector $f^{\mathrm{Up}}(\underline{E}, c) \in \mathbb{R}^{N}$ defined by

$$
f^{\mathrm{Up}}(\underline{E}, c)=\sum_{l=1}^{r} x^{l}
$$

where $x^{r}=f\left(E_{r}, c^{r}\right)$ and, for $l=r-1, \ldots, 1, x^{l}$ is recursively defined by

$$
x^{l}=f\left(E_{l}, c^{l}-\sum_{\lambda=l+1}^{r} x^{\lambda}\right) .
$$

Our assumption on sequential bankruptcy problems and the boundedness constraints of bankruptcy rules ensure that all the problems above are bankruptcy problems.

Following the idea behind the upwards mechanism, a rule $f$ is an upwards sequential bankruptcy rule or upwards rule if for every $(\underline{E}, c) \in \mathcal{B}^{N_{1}, \ldots, N_{r}}, x^{r+1}=\mathbf{0}$ and $x^{k}$ recursively 


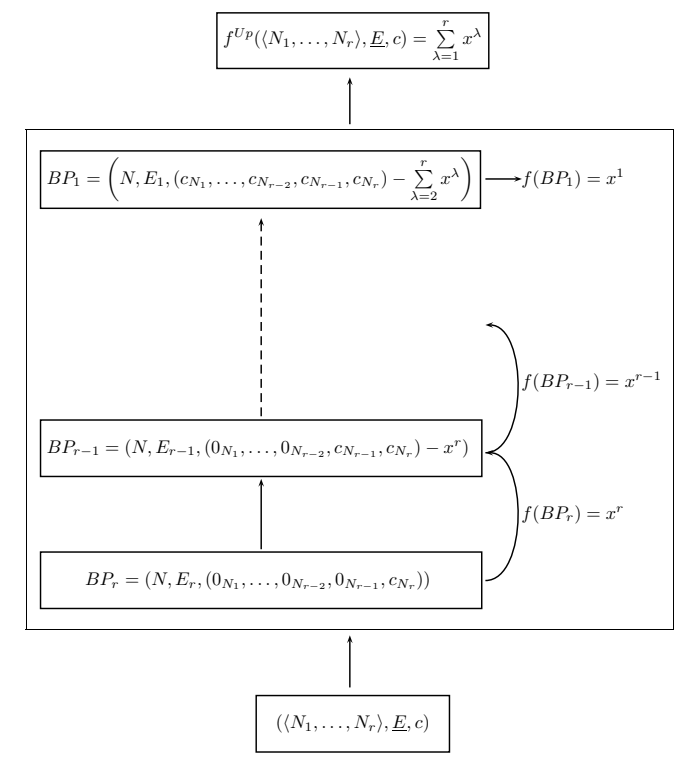

Figure 3: Summary of the upwards mechanism.

defined by

$$
x^{k}=f\left(\underline{E_{k}}, c^{k}-\sum_{\lambda=k+1}^{r+1} x^{\lambda}\right)
$$

for $k=r, r-1, \ldots, 2,1$, imply

$$
f(\underline{E}, c)=\sum_{\lambda=1}^{r} x^{\lambda}
$$

That is, if $f$ is an upwards rule, it is the same to directly apply $f$ to $(\underline{E}, c)$, or to apply the rule to $\left(\underline{E}^{r}, c^{r}\right)$ and, after updating the claims, work all the way up to $\left(\underline{E}-\underline{E}^{2}, d^{1}\right)$, where $d^{1}$ represents the updated claims. Clearly, the upwards mechanism generates upwards rules.

The following result states that the properties of invariance under claims truncation, equal treatment of equals, and minimal rights first in the class of bankruptcy problems are inherited in the class of sequential bankruptcy problems by the upwards mechanism. The proof is postponed to the appendix.

Proposition 3.2. Let $f$ be a bankruptcy rule.

(i) If $f$ satisfies invariance under claims truncation, then, $f^{U p}$ satisfies invariance under claims truncation.

(ii) If $f$ satisfies invariance under claims truncation, then, $f^{U p}$ satisfies weak invariance under claims truncation.

(iii) If $f$ satisfies equal treatment of equals, then, $f^{\text {Up }}$ satisfies equal treatment of equals.

(iv) If $f$ satisfies minimal rights first, then, $f^{U p}$ satisfies weak minimal rights first.

Next, we turn our attention to the properties of duality (cf. Aumann and Maschler 1985), 
composition (cf. Young 1988), and path independence (cf. Moulin 1987) ${ }^{1}$. For this, we need to introduce new notation. Let $(\underline{E}, c) \in \mathcal{B}^{N_{1}, \ldots, N_{r}}$. For $l \in\{1, \ldots, r\}$, let

$$
F_{\lambda}^{l,(\underline{E}, c)}= \begin{cases}\sum_{\mu=l}^{r} L_{\mu} & \text { if } \lambda=l \\ 0 & \text { otherwise. }\end{cases}
$$

By assumption, $F_{l}^{l,(\underline{E}, c)} \geq 0$ and $\left(F_{1}^{l,(\underline{E}, c)}, \ldots, F_{r}^{l,(\underline{E}, c)}, c\right) \in \mathcal{B}^{N_{1}, \ldots, N_{r}}$ for all $l \in\{1, \ldots, r\}$. If no confusion is to be expected, we write $F_{\lambda}^{l}$ instead of $F_{\lambda}^{l,(\underline{E}, c)}$. Let $\underline{F}^{l}=\left(F_{1}^{l}, \ldots, F_{r}^{l}\right)$. The sequential bankruptcy problem $\left(\underline{F}^{l}, c\right)$ represents the problem of sharing the sum of the aggregate loss in groups $N_{l}, \ldots, N_{r}$ among their members.

The rule $f^{\mathrm{UpD}}$ is the upwards dual rule of $f$ if for each $(\underline{E}, c) \in \mathcal{B}^{N_{1}, \ldots, N_{r}}$,

$$
f^{\mathrm{UpD}}(\underline{E}, c)=c-f\left(\underline{F}^{1}, c-\left(c^{2}-x^{2}\right)\right)
$$

where $c^{r+1}=x^{r+1}=\mathbf{0}$ and for $k=r, r-1, \ldots, 1$,

$$
x^{k}=f\left(\underline{F}^{k}, c^{k}-\left(c^{k+1}-x^{k+1}\right)\right) .
$$

The rule $f$ is upwards self-dual if $f=f^{\mathrm{UpD}}$.

Remark 3.1. Let $f^{\mathrm{UpD}}$ be the upwards dual rule of $f$ and let $(\underline{E}, c) \in \mathcal{B}^{N_{1}, \ldots, N_{r}}$ with $E_{1}=\ldots=$ $E_{k-1}=0$ and $E_{k}>0$ for some $k \in\{2, \ldots, r\}$. Using that $f^{\mathrm{UpD}}(\underline{E}, c)=\sum_{l=1}^{r}\left(c^{l}-x^{l}-\left(c^{l+1}-\right.\right.$ $\left.x^{l+1}\right)$ ), it readily follows that

$$
f^{\mathrm{UpD}}(\underline{E}, c)=c^{k}-x^{k}
$$

The following result establishes the one-to-one relation between an upwards rule and its dual. Besides, it shows that given two dual bankruptcy rules, the corresponding upwards rules obtained with the upwards mechanism are also upwards dual. The proof is postponed to the appendix.

\section{Lemma 3.3.}

(i) Let $f$ be an upwards rule and let $f^{U p D}$ be its upwards dual rule. Then, $f^{U p D}$ is also an upwards rule and $f$ is the upwards dual rule of $f^{\mathrm{UpD}}$.

(ii) Let $f$ and $g$ be two dual bankruptcy rules. Then, $f^{U p}$ and $g^{U p}$ are upwards dual.

A rule $f$ satisfies upwards composition if for each $(\underline{E}, c) \in \mathcal{B}^{N_{1}, \ldots, N_{r}}$, each $k \in\{1, \ldots, r\}$, and each $E_{k}^{\prime} \in \mathbb{R}_{+}$with $E_{k}^{\prime} \leq E_{k}, x^{k}=f\left(\underline{E_{k}^{\prime}}+\underline{E}^{k+1}, c^{k}\right)$ implies

$$
f(\underline{E}, c)=f\left(\underline{E}-\underline{E_{k}^{\prime}}-\underline{E}^{k+1}, c-x^{k}\right)+f\left(\underline{E_{k}^{\prime}}+\underline{E}^{k+1}, c^{k}\right) .
$$

Lemma 3.4. Any rule satisfying upwards composition is an upwards rule.

\footnotetext{
${ }^{1}$ We follow here the nomenclature in Herrero and Villar (2001).
} 
Proof. Let $f$ satisfy upwards composition and let $(\underline{E}, c) \in \mathcal{B}^{N_{1}, \ldots, N_{r}}$. Let $E_{r}^{\prime}=E_{r}$ and $x^{r}=$ $f\left(\underline{E_{r}}, c^{r}\right)$. By upwards composition,

$$
f(\underline{E}, c)=f\left(\underline{E}-\underline{E_{r}}, c-x^{r}\right)+f\left(\underline{E_{r}}, c^{r}\right) .
$$

Let $E_{k}^{\prime}=E_{k}$ and $x^{k}=f\left(\underline{E_{k}}, c^{k}-\sum_{\lambda=k+1}^{r} x^{\lambda}\right)$ for $k=r-1, \ldots, 1$. Reiteratively applying upwards composition, we get

$$
f(\underline{E}, c)=\sum_{\lambda=1}^{r} x^{l}
$$

The following result states that composition in the class of bankruptcy problems is inherited in the class of sequential bankruptcy problems by the upwards mechanism. The proof is postponed to the appendix.

Proposition 3.5. Let $f$ be a bankruptcy rule satisfying composition, then, $f^{U p}$ satisfies upwards composition.

Theorem 3.6. The upwards constrained equal awards rule is the only rule satisfying equal treatment of equals, weak invariance under claims truncation, and upwards composition.

Proof. It is well established that the constrained equal awards rule for bankruptcy problems satisfies equal treatment of equals, invariance under claims truncation, and composition. By Propositions 3.2 and 3.5, the upwards constrained equal awards rule satisfies equal treatment of equals, weak invariance under claims truncation, and upwards composition as well. We now prove uniqueness. Let $f$ be a rule satisfying equal treatment of equals, weak invariance under claims truncation, and upwards composition. By Lemma 3.4, $f$ is an upwards rule. Next, we show $f=\mathrm{CEA}^{\mathrm{Up}}$. Let $(\underline{E}, c) \in \mathcal{B}^{N_{1}, \ldots, N_{r}}$. By upwards composition, $x^{r}=f\left(\underline{E_{r}}, c^{r}\right)$ implies

$$
f(\underline{E}, c)=f\left(\underline{E}-\underline{E_{r}}, c-x^{r}\right)+f\left(\underline{E_{r}}, c^{r}\right) .
$$

Following the same lines as in Dagan (1996), it is readily seen that equal treatment of equals, weak invariance under claims truncation, and upwards composition imply $f\left(\underline{E_{r}}, c^{r}\right)=\mathrm{CEA}^{\mathrm{Up}}\left(\underline{E_{r}}, c^{r}\right)$ and

$$
f(\underline{E}, c)=f\left(\underline{E}-\underline{E}_{r}, c-x^{r}\right)+\mathrm{CEA}^{\mathrm{Up}}\left(\underline{E_{r}}, c^{r}\right)
$$

with $x^{r}=\mathrm{CEA}^{\mathrm{Up}}\left(\underline{E_{r}}, c\right)$. Recursively, following the same lines as above, for $k \in\{1, \ldots, r-1\}$, $x^{r}=f\left(\underline{E_{r}}, c^{r}\right)=\mathrm{CEA}^{\mathrm{Up}}\left(\underline{E_{r}}, c^{r}\right)$ and $x^{l}=f\left(\underline{E_{l}}, c^{l}-\sum_{\lambda=l+1}^{r} x^{\lambda}\right)=\mathrm{CEA}^{\mathrm{Up}}\left(\underline{E_{l}}, c^{l}-\sum_{\lambda=l+1}^{r} x^{\lambda}\right)$ for $l \in\{k, \ldots, r-1\}$, imply

$$
\begin{aligned}
f(\underline{E}, c) & =f\left(\underline{E}-\underline{E^{k}}, c-\sum_{\mu=\lambda+1}^{r} x^{\mu}\right)+\sum_{\lambda=k}^{r} f\left(\underline{E_{\lambda}}, c^{\lambda}-\sum_{\mu=\lambda+1}^{r} x^{\mu}\right) \\
& =f\left(\underline{E}-\underline{E^{k}}, c-\sum_{\mu=\lambda+1}^{r} x^{\mu}\right)+\sum_{\lambda=k}^{r} \mathrm{CEA}^{\mathrm{Up}}\left(\underline{E_{\lambda}}, c^{\lambda}-\sum_{\mu=\lambda+1}^{r} x^{\mu}\right)
\end{aligned}
$$


Therefore, for $k=1$,

$$
f(\underline{E}, c)=\sum_{\lambda=1}^{r} \mathrm{CEA}^{\mathrm{Up}}\left(\underline{E_{\lambda}}, c^{\lambda}-\sum_{\mu=\lambda+1}^{r} x^{\mu}\right)=\mathrm{CEA}^{\mathrm{Up}}(\underline{E}, c)
$$

As an immediate consequence of Proposition 3.2 and Theorem 3.6, we have

Corollary 3.7. The upwards constrained equal awards rule is the only rule satisfying equal treatment of equals, invariance under claims truncation, and upwards composition.

A rule $f$ satisfies upwards path independence if for each $(\underline{E}, c) \in \mathcal{B}^{N_{1}, \ldots, N_{r}}$, each $k \in\{1, \ldots, r\}$, and each $E_{k}^{\prime} \in \mathbb{R}_{+}$with $E_{k}^{\prime} \leq E_{k}, d^{k}=f\left(\underline{E}^{k}, c^{k}\right), x^{k+1}=f\left(\underline{E}^{k+1}, c^{k+1}\right)$, and $x^{\prime k}=f\left(\underline{E_{k}^{\prime}}, d^{k}-x^{k+1}\right)$, imply

$$
\begin{aligned}
f\left(\underline{E}-\underline{E_{k}}+\underline{E_{k^{\prime}}^{\prime}} c\right)= & f\left(\underline{E}-\underline{E_{k}}-\underline{E}^{k+1}, c-x^{\prime k}-x^{k+1}\right)+f\left(\underline{E}_{k^{\prime}}^{\prime} d^{k}-x^{k+1}\right) \\
& +f\left(\underline{E}^{k+1}, c^{k+1}\right) .
\end{aligned}
$$

Following the same lines as Lemma 3.4, one can see that path independence implies being an upwards rule. The proof is, therefore, omitted.

Lemma 3.8. Any rule satisfying upwards path independence is an upwards rule.

Following Herrero and Villar (2001), two properties $\mathcal{P}$ and $\mathcal{P}^{\mathrm{UpD}}$ are upwards dual when a rule $f$ satisfies $\mathcal{P}$ if, and only if, its upwards dual rule $f^{\mathrm{UpD}}$ satisfies $\mathcal{P}^{\mathrm{UpD}}$. A property $\mathcal{P}$ is upwards self-dual when a rule $f$ satisfies $\mathcal{P}$ if, and only if, its upwards dual rule $f^{\mathrm{UpD}}$ satisfies $\mathcal{P}$ as well.

The proof of the following result follows the same lines as the proof of Theorem 0 in Herrero and Villar (2001). It is, therefore, omitted.

Theorem 3.9 (Herrero and Villar, 2001). Let a rule $f$ be characterized by a set of independent properties $\mathcal{P}_{1}, \ldots, \mathcal{P}_{s}$. Let $\mathcal{P}_{1}^{U p D}, \ldots, \mathcal{P}_{s}^{U p D}$ be the dual properties of $\mathcal{P}_{1}, \ldots, \mathcal{P}_{s}$, respectively. Then, the upwards dual rule $f^{U p D}$ is characterized by $\mathcal{P}_{1}^{U p D}, \ldots, \mathcal{P}_{s}^{U p D}$ and these properties are also independent.

The following result states that upwards composition and upwards path independence are upwards dual properties, and weak minimal rights first and weak invariance under claims truncation are upwards dual properties as well. The proof of these results follow the same lines as their counterparts in the framework of bankruptcy problems and is, therefore, omitted.

\section{Lemma 3.10.}

(i) Upwards composition and upwards path independence are upwards dual.

(ii) Weak minimal rights first and weak invariance under claims truncation are upwards dual.

(iii) Equal treatment of equals is upwards self-dual.

The following result is a direct consequence of Lemma 3.3 (ii), Theorems 3.6 and 3.9, and Lemma 3.10. 
Theorem 3.11. The upwards constrained equal losses rule is the only rule satisfying equal treatment of equals, weak minimal rights first, and upwards path independence.

To conclude this section, we characterize the upwards proportional rule. For this, we need to introduce one last property. Let $f$ be a rule. Let $c \in \mathbb{R}^{N_{1} \cup \ldots \cup N_{r}}$. For $k \in\{1, \ldots, r\}$, let $p_{f}^{k, c}:\left[0, \sum_{l=k}^{r} c\left(N_{l}\right)\right] \rightarrow \mathbb{R}^{N}$ be defined by $p_{f}^{k, c}\left(E_{k}\right)=f\left(\underline{E_{k}}, c\right)$. We say that $f$ is weak continuous if for each $c \in \mathbb{R}^{N_{1} \cup \ldots \cup N_{r}}$ and each $k \in\{1, \ldots, r\}, p_{f}^{k, c}$ is continuous.

As remarked in Herrero and Villar (2001), Young's characterization of the proportional rule uses continuity. However, only continuity with respect to the estate is needed. In our case, we only need the requirement of weak continuity, which follows from both upwards composition and upwards path independence. The proof of the following result follows the same lines as the proof of Theorem 3.6 and is, therefore, omitted.

\section{Theorem 3.12.}

(i) The upwards proportional rule is the only rule satisfying upwards composition and upwards selfduality.

(ii) The upwards proportional rule is the only rule satisfying upwards path independence and upwards self-duality.

\section{Downwards and two-steps mechanisms}

Here, we introduce the downwards and two-steps mechanisms to generalize bankruptcy rules to sequential bankruptcy rules. The downwards mechanism was introduced in Ansink and Weikard (2012) for the special case with $\left|N_{1}\right|=\ldots=\left|N_{r}\right|=1$. The two-steps mechanism is related to the two-step procedure for bankruptcy problems with a priori unions in Borm et al. (2005). Before giving the formal description, we outline them by means of an example.

Example 4.1. Reconsider the problem of sharing water in a river in Example 2.1 (see Figure 1). As pointed out in Example 2.3, we can model this problem as a sequential bankruptcy problem with $N=$ $\{A, B, C, D, E, F\}, N_{1}=\{A, B\}, N_{2}=\{C, D, E\}$, and $N_{3}=\{F\} ; E_{1}=6, E_{2}=5$, and $E_{3}=3$; and $c=(2,3,1,2,3,5)$.

To allocate the available water among the claimants, we will use the constrained equal awards rule. We start explaining the downwards mechanism. First, recall that the water inflow $E_{1}$ can be shared among all the claimants since water flows downstream. Therefore, agents in $N_{2}$ and $N_{3}$ claim $c_{C}+c_{D}+$ $c_{E}+c_{F}-E_{2}-E_{3}=L_{2}+L_{3}=3$. We can apply the constrained equal awards rule to $\left(E_{1},\left(c_{N_{1}}, 3\right)\right)$ and set $x^{1}=\operatorname{CEA}(6,(2,3,3))=(2,2,2)$. Second, the water inflow $E_{2}+2$ can be shared among the claimants in $N_{2}$ and $N_{3}$ since water flows downstream. Therefore, the agent in $N_{3}$ claims $c_{F}-$ $E_{3}=L_{3}=2$. We can now apply the constrained equal awards rule to $\left(E_{2}+2,\left(c_{N_{2}}, 2\right)\right)$ and set $x^{2}=\operatorname{CEA}(7,(1,2,3,2))=(1,2,2,2)$. Third and last, the water inflow $E_{3}+2$ can be used only by the claimant in $N_{3}$. Then, $x^{3}=C E A(3+2,(5))=(5)$. The downwards mechanism applied to the constrained equal awards rule leads to the allocation $(2,2,1,2,2,5)$. 
Next, we use the two-steps mechanism. First, we associate a bankruptcy problem where the agents are the groups: $\{1,2,3\}$, the estate is the total estate: $E=E_{1}+E_{2}+E_{3}=14$; and the claim of group $l \in\{1,2,3\}$ is its total claim truncated to the total estate over which they claim: $d_{l}=$ $\min \left\{c\left(N_{l}\right), \sum_{\lambda=1}^{l} E_{\lambda}\right\}$. Then, $d=(5,6,5)$. We set $x=\operatorname{CEA}(14,(5,6,5))=\left(4 \frac{2}{3}, 4 \frac{2}{3}, 4 \frac{2}{3}\right)$. Second, we apply the constrained equal awards rule to share each group's allocation among its members. The two-steps mechanism applied to the constrained equal awards rule leads to the allocation:

$$
\left(\left(4 \frac{2}{3},(2,3)\right), C E A\left(4 \frac{2}{3},(1,2,3)\right), C E A\left(4 \frac{2}{3},(5)\right)\right)=\left(2,2 \frac{2}{3}, 1,1 \frac{5}{6}, 1 \frac{5}{6}, 4 \frac{2}{3}\right) .
$$

Next, we formally describe both mechanisms. We start with the downwards mechanism. For this, given $(\underline{E}, c) \in \mathcal{B}^{N_{1}, \ldots, N_{r}}$ and $l \in\{1, \ldots, r-1\}$, we denote by $i_{l+1}$ the fictitious claimant that represents the members in $N_{l+1} \cup \ldots \cup N_{r}$ with claim $\sum_{\lambda=l+1}^{r} L_{\lambda}$ over $E_{l}$.

Given a bankruptcy rule $f$, the downwards mechanism generates a rule for sequential bankruptcy problems, $f^{\mathrm{D}}$, that assigns to each $(\underline{E}, c) \in \mathcal{B}^{N_{1}, \ldots, N_{r}}$, a vector $f^{\mathrm{D}}(\underline{E}, c) \in \mathbb{R}^{N}$ defined by

$$
f^{\mathrm{D}}(\underline{E}, c)=\left(x_{N_{1}}^{1}, \ldots, x_{N_{r}}^{r}\right)
$$

where $x^{0}=0$, for $l=1, \ldots, r-1, x^{l}$ is recursively defined by

$$
x^{l}=f\left(E_{l}+x_{i_{l}}^{l-1},\left(c_{N_{l}}, \sum_{\lambda=l+1}^{r} L_{\lambda}\right)\right),
$$

and $x^{r}=f\left(E_{r}+x_{i_{r}}^{r-1}, c_{N_{r}}\right)$.

To conclude, we describe the two-steps mechanism. Given a bankruptcy rule $f$, the twosteps mechanism generates a rule for sequential bankruptcy problems, $f^{2 \mathrm{~s}}$, that assigns to each $(\underline{E}, c) \in \mathcal{B}^{N_{1}, \ldots, N_{r}}$, a vector $f^{2 \mathrm{~s}}(\underline{E}, c) \in \mathbb{R}^{N}$ defined by

$$
f^{2 \mathrm{~s}}(\underline{E}, c)=\left(f\left(x_{1}, c_{N_{1}}\right), \ldots, f\left(x_{r}, c_{N_{r}}\right)\right)
$$

where

$$
x=f\left(\sum_{\lambda=1}^{r} E_{\lambda},\left(d_{1}, \ldots, d_{r}\right)\right),
$$

with $d_{l}=\min \left\{c\left(N_{l}\right), \sum_{\lambda=1}^{l} E_{\lambda}\right\}$ for $l \in\{1, \ldots, r\}$.

Our assumption on sequential bankruptcy problems and the boundedness condition on bankruptcy rules ensure that all the problems in the description of both mechanisms are bankruptcy problems.

Composition and path independence are not inherited in the class of sequential bankruptcy problems by the downwards and two-steps mechanisms in the form of upwards composition and upwards path independence since they do not generate upwards rules. Moreover, equal 


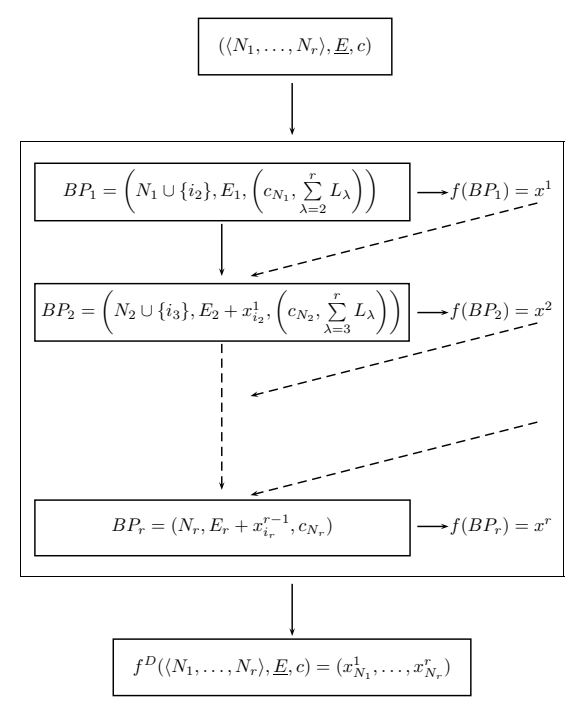

Downwards mechanism.

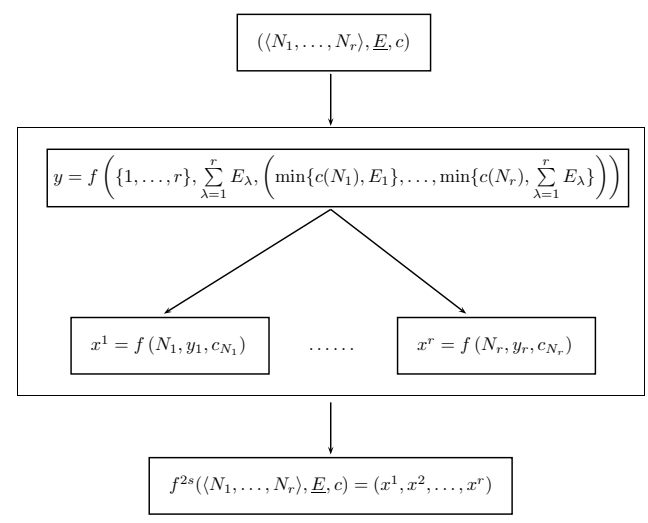

Two-steps mechanism.

Figure 4: Summary of the two mechanisms.

treatment of equals need not be inherited in the class of sequential bankruptcy problems by the downwards and two-steps mechanisms, as the following example illustrates.

Example 4.2. Let $\left(\left\langle N_{1}, N_{2}, N_{3}\right\rangle,\left(E_{1}, E_{2}, E_{3}\right), c\right)$ be the sequential bankruptcy problem with $N_{1}=\{1\}$, $N_{2}=\{2,3\}$, and $N_{3}=\{4,5\}, E_{1}=0, E_{2}=9$, and $E_{3}=0, c=(1,3,4,1,3)$. We consider the constrained equal awards rule, which satisfies equal treatment of equals. In our sequential bankruptcy problem, agents 2 and 5 are "equal". However, $C E A^{D}$ and $C E A^{2 s}$ do not assign them the same amount since $C E A^{D}(\underline{E}, c)=(0,3,3,1,2)$ and $C E A^{2 s}(\underline{E}, c)=\left(0,2 \frac{1}{2}, 2 \frac{1}{2}, 1,3\right)$. Therefore, neither $C E A^{D}$, nor $C E A^{2 s}$ satisfy equal treatment of equals.

To conclude, it turns out that the properties of invariance under claims truncation and minimal rights first in the class of bankruptcy problems are inherited in the class of sequential bankruptcy problems by the downwards and the two-steps mechanisms. The proof is postponed to the appendix.

Proposition 4.3. Let $f$ be a bankruptcy rule.

(i) If $f$ satisfies invariance under claims truncation, then, $f^{D}$ and $f^{2 s}$ satisfy invariance under claims truncation.

(ii) If $f$ satisfies invariance under claims truncation, then, $f^{D}$ and $f^{2 s}$ satisfy weak invariance under claims truncation.

(iii) If $f$ satisfies minimal rights first, then, $f^{D}$ and $f^{2 s}$ satisfy weak minimal rights first. 


\section{A Appendix}

\section{Proof of Proposition 3.1.}

(i) Let $(\underline{E}, c) \in \mathcal{B}^{N_{1}, \ldots, N_{r}}$. Since $f$ satisfies invariance under claims truncation,

$$
x^{r}=f\left(E_{r}, c^{r}\right)=f\left(E_{r},\left(\min \left\{c_{i}^{r}, \sum_{\lambda=1}^{r} E_{\lambda}\right\}\right)_{i \in N}\right)=f\left(E_{r},\left(c^{\underline{E}}\right)^{r}\right)=y^{r}
$$

Applying again invariance under claims truncation,

$$
\begin{aligned}
x^{r-1} & =f\left(E_{r-1}, c^{r-1}-x^{r}\right) \\
& =f\left(E_{r-1},\left(\left(\min \left\{c_{i}^{r-1}-x_{i}^{r}, \sum_{\lambda=1}^{r-1} E_{\lambda}\right\}\right)_{i \in N \backslash N_{r}}\left(\left(\min \left\{c_{i}^{r-1}-x_{i}^{r}, \sum_{\lambda=1}^{r} E_{\lambda}\right\}\right)_{i \in N_{r}}\right)\right)\right. \\
& =f\left(E_{r-1},\left(\left(\min \left\{c_{i}^{r-1}, \sum_{\lambda=1}^{r-1} E_{\lambda}+x_{i}^{r}\right\}\right)_{i \in N \backslash N_{r}},\left(\min \left\{c_{i}^{r-1}, \sum_{\lambda=1}^{r} E_{\lambda}+x_{i}^{r}\right\}\right)_{i \in N_{r}}\right)-x^{r}\right) \\
& =f\left(E_{r-1},\left(\left(\min \left\{c_{i}^{r-1}, \sum_{\lambda=1}^{r-1} E_{\lambda}\right\}\right)_{i \in N \backslash N_{r}},\left(\min \left\{c_{i}^{r-1}, \sum_{\lambda=1}^{r} E_{\lambda}\right\}\right)_{i \in N_{r}}\right)-x^{r}\right) \\
& =f\left(E_{r-1},(c \underline{E})^{r-1}-y^{r}\right)=y^{r-1}
\end{aligned}
$$

where the fourth equality is a direct consequence of independence under claims truncation since $E_{r-1} \leq \sum_{\lambda=1}^{r} E_{\lambda} \leq \sum_{\lambda=1}^{r} E_{\lambda}+x_{i}^{r}$ and the fifth equality follows since for each $i \in N \backslash$ $\left(N_{r-1} \cup N_{r}\right), c_{i}^{r-1}=0=\min \left\{c_{i}^{r-1}, \sum_{\lambda=1}^{r-1} E_{\lambda}\right\}=\left(c_{i}^{\underline{E}}\right)^{r-1}$. Reiteratively applying invariance under claims truncation of $f$, we get $x^{l}=f\left(E_{l}, c^{l}-\sum_{\lambda=l+1}^{r} x^{\lambda}\right)=f\left(E_{l},\left(c^{\underline{E}}\right)^{l}-\sum_{\lambda=l+1}^{r} y^{\lambda}\right)=y^{l}$ for $l \in\{1, \ldots, r-2\}$ and

$$
f^{\mathrm{Up}}(\underline{E}, c)=\sum_{\lambda=1}^{r} x^{l}=\sum_{\lambda=1}^{r} y^{l}=f^{\mathrm{Up}}\left(\underline{E}, c^{\underline{E}}\right) .
$$

(ii) By (i), $f^{\mathrm{Up}}$ satisfies invariance under claims truncation and, therefore, weak invariance under claims truncation.

(iii) Let $(\underline{E}, c) \in \mathcal{B}^{N_{1}, \ldots, N_{r}}$ and $k, l \in\{1, \ldots, r\}, k \leq l$, with $\sum_{\lambda=1}^{k} E_{\lambda}=\sum_{\lambda=1}^{l} E_{\lambda}$, and let $i \in N_{k}$ and $j \in N_{l}$ with $c_{i}=c_{j}$. Obviously, $E_{k+1}=\ldots=E_{l}=0$. Let $f^{\mathrm{Up}}(\underline{E}, c)=\sum_{l=1}^{r} x^{l}$ with $x^{l}, l=1, \ldots, r$ as in the description of the upwards mechanism. Then, $x_{i}^{\lambda}=x_{j}^{\lambda}=0$ for $\lambda=k+1, \ldots, r+1$. Therefore,

$$
c_{i}-\sum_{\lambda=k+1}^{r+1} x_{i}^{\lambda}=c_{i}=c_{j}=c_{j}-\sum_{\lambda=k+1}^{r+1} x_{j}^{\lambda}
$$

and, applying equal treatment of equals,

$$
x_{i}^{k}=f_{i}\left(E_{k}, c^{k}-\sum_{\lambda=k+1}^{r+1} x^{\lambda}\right)=f_{j}\left(E_{k}, c^{k}-\sum_{\lambda=k+1}^{r+1} x^{\lambda}\right)=x_{j}^{k} .
$$


Reiteratively applying equal treatment of equals of $f$, we get $x_{i}^{l}=x_{j}^{l}$ for $l \in\{1, \ldots, r\}$ and

$$
f_{i}^{\mathrm{Up}}(\underline{E}, c)=\sum_{\lambda=1}^{r} x_{i}^{l}=\sum_{\lambda=1}^{r} x_{j}^{l}=f_{j}^{\mathrm{Up}}(\underline{E}, c)
$$

(iv) Let $k \in\{1, \ldots, r\}$ and let $\left(\underline{E_{k}}, c\right) \in \mathcal{B}^{N_{1}, \ldots, N_{r}}$. Then, by Proposition 2.1 and the assumption on sequential bankruptcy problems, $m_{i}=0$ for $i \in N_{l}, l \in\{1, \ldots, k-1\}$. Moreover, for $i \in N_{l}$, $l \in\{k, \ldots, r\}$,

$$
\begin{aligned}
m_{i} & =\max _{1 \leq \lambda \leq l}\left\{\left(c_{i}-\sum_{\mu=\lambda}^{r} L_{\mu}\right)_{+}\right\} \\
& =\max \left\{\max _{1 \leq \lambda \leq k}\left\{\left(c_{i}-\left(\sum_{\mu=\lambda}^{r} c\left(N_{\mu}\right)-E_{k}\right)\right)_{+}\right\}^{\prime} \max _{k+1 \leq \lambda \leq l}\left\{\left(c_{i}-\sum_{\mu=\lambda}^{r} c\left(N_{\mu}\right)\right)_{+}\right\}\right\} \\
& =\left(c_{i}-\left(\sum_{\mu=k}^{r} c\left(N_{\mu}\right)-E_{k}\right)\right)_{+}
\end{aligned}
$$

where the last equality is a direct consequence of $c \in \mathbb{R}_{+}^{N}$. Therefore, $m=m\left(E_{k}, c^{k}\right)$ and, applying minimal rights first to $f$,

$$
\begin{aligned}
f^{\mathrm{Up}}\left(\underline{E_{k}}, c\right) & =f\left(E_{k}, c^{k}\right)=m\left(E_{k}, c^{k}\right)+f\left(E_{k}-\sum_{i \in N} m_{i}\left(E_{k}, c^{k}\right), c^{k}-m\left(E_{k}, c^{k}\right)\right) \\
& =m+f^{\mathrm{Up}}\left(0, \ldots, 0, E_{k}-\sum_{\lambda=k}^{r} \sum_{i \in N_{\lambda}} m_{i}, 0, \ldots, 0, c-m\right) .
\end{aligned}
$$

\section{Proof of Lemma 3.3.}

(i) First, we show that $f^{\mathrm{UpD}}$ is an upwards rule. Let $(\underline{E}, c) \in \mathcal{B}^{N_{1}, \ldots, N_{r}}$.

$$
f^{\mathrm{UpD}}(\underline{E}, c)=c-f\left(\underline{F}^{1}, c-\left(c^{2}-x^{2}\right)\right)
$$

with $x^{l}, l=1, \ldots, r$, as in the definition of upwards duality. Let $y^{r+1}=\mathbf{0}$ and for $l=r, r-$ $1, \ldots, 2,1$, let $y^{l}$ be recursively defined by

$$
y^{l}=f^{\mathrm{UpD}}\left(\underline{E_{l}}, c^{l}-\sum_{\lambda=l+1}^{r+1} y^{\lambda}\right) .
$$

We show by induction that $c^{l}-x^{l}=\sum_{\lambda=l}^{r} y^{\lambda}$ for every $l=r, r-1, \ldots, 1$. For $l=r$, and using Remark 3.2,

$$
y^{r}=f^{\mathrm{UpD}}\left(\underline{E_{r}}, c^{r}\right)=c^{r}-f\left(\underline{L_{r}}, c^{r}\right)=c^{r}-f\left(\underline{F}^{r}, c^{r}\right)=c^{r}-x^{r}
$$


Assume now that $c^{l+1}-x^{l+1}=\sum_{\lambda=l+1}^{r} y^{\lambda}$ for some $l \in\{1, \ldots, r-1\}$. Then,

$$
\begin{aligned}
\sum_{\lambda=l}^{r} y^{\lambda} & =f^{\mathrm{UpD}}\left(\underline{E_{l}}, c^{l}-\sum_{\lambda=l+1}^{r} y^{\lambda}\right)+\sum_{\lambda=l+1}^{r} y^{\lambda} \\
& =c^{l}-\sum_{\lambda=l+1}^{r} y^{\lambda}-f\left(\underline{F}^{l}, c^{l}-\sum_{\lambda=l+1}^{r} y^{\lambda}\right)+\sum_{\lambda=l+1}^{r} y^{\lambda} \\
& =c^{l}-f\left(\underline{F}^{l}, c^{l}-\sum_{\lambda=l+1}^{r} y^{\lambda}\right) \\
& =c^{l}-f\left(\underline{F}^{l}, c^{l}-\left(c^{l+1}-x^{l+1}\right)\right) \\
& =c^{l}-x^{l}
\end{aligned}
$$

where the second equality follows since $f^{\mathrm{UpD}}$ is the upwards dual rule of $f$ and by Remark 3.2. Then,

$$
\sum_{\lambda=1}^{r} y^{\lambda}=c^{1}-x^{1}=c^{1}-f\left(\underline{F}^{1}, c^{1}-\left(c^{2}-x^{2}\right)\right)=f^{\mathrm{UpD}}(\underline{E}, c) .
$$

Second, we show that $f$ is the upwards dual rule of $f^{\mathrm{UpD}}$. Let $f(\underline{E}, c)=\sum_{\lambda=1}^{r} \bar{y}^{\lambda}$ with $\bar{y}^{l}, l=$ $1, \ldots, r$, as in the definition of upwards rule. Let $c^{r+1}=\bar{x}^{r+1}=0$ and $\bar{x}^{l}=f^{\mathrm{UpD}}\left(\underline{F}^{l}, c^{l}-\left(c^{l+1}-\bar{x}^{l+1}\right)\right)$ for $l=r, r-1, \ldots, 1$. We show by induction that $c^{l}-\bar{x}^{l}=\sum_{\lambda=l}^{r} \bar{y}^{\lambda}$ for every $l=r, r-1, \ldots, 1$. For $l=r$, and using Remark 3.2,

$$
c^{r}-\bar{x}^{r}=c^{r}-f^{\mathrm{UpD}}\left(\underline{F}^{r}, c^{r}\right)=c^{r}-\left(c^{r}-f\left(\underline{E}, c^{r}\right)\right)=f\left(\underline{E_{r}}, c^{r}\right)=\bar{y}^{r} .
$$

Assume now that $c^{l+1}-\bar{x}^{l+1}=\sum_{\lambda=l+1}^{r} \bar{y}^{\lambda}$ for some $l \in\{1, \ldots, r-1\}$. Then,

$$
\begin{aligned}
c^{l}-\bar{x}^{l} & =c^{l}-f^{\mathrm{UpD}}\left(\underline{F}^{l}, c^{l}-\left(c^{l+1}-\bar{x}^{l+1}\right)\right) \\
& =c^{l}-\left(c^{l}-\left(c^{l+1}-\bar{x}^{l+1}\right)-f\left(\underline{E}_{l}, c^{l}-\left(c^{l+1}-\bar{x}^{l+1}\right)\right)\right) \\
& =c^{l+1}-\bar{x}^{l+1}+f\left(\underline{E_{l}}, c^{l}-\left(c^{l+1}-\bar{x}^{l+1}\right)\right) \\
& =\sum_{\lambda=l+1}^{r} \bar{y}^{\lambda}+f\left(\underline{E_{l}}, c^{l}-\sum_{\lambda=l+1}^{r} \bar{y}^{\lambda}\right)=\sum_{\lambda=l}^{r} \bar{y}^{\lambda}
\end{aligned}
$$

where the second equality follows since $f^{\mathrm{UpD}}$ is the upwards dual rule of $f$ and by Remark 3.2. Then,

$$
c-f^{\mathrm{UpD}}\left(\underline{F}^{1}, c-\left(c^{2}-\bar{x}^{2}\right)\right)=c-\bar{x}^{1}=\sum_{\lambda=1}^{r} \bar{y}^{\lambda}=f(\underline{E}, c) .
$$


(ii) Since $f^{\mathrm{Up}}$ and $g^{\mathrm{Up}}$ are upwards rules, it suffices to show that $g^{\mathrm{Up}}$ is the upwards dual rule of $f^{\mathrm{Up}}$ by (i). Let $(\underline{E}, c) \in \mathcal{B}^{N_{1}, \ldots, N_{r}}$. Let $g^{\mathrm{Up}}(\underline{E}, c)=\sum_{\lambda=1}^{r} x^{\lambda}$ with $x^{l}, l=1, \ldots, r$, as in the definition of upwards rule. Moreover, for $l=r, r-1, \ldots, 1$, let $y^{l}=f\left(\sum_{\lambda=l}^{r} L_{\lambda}, c^{l}-\left(c^{l+1}-y^{l+1}\right)\right)$ as in the definition of upwards dual rule. We show by induction that for $l=r, r-1, \ldots, 1, x^{l}=$ $c^{l}-y^{l}-\left(c^{l+1}-y^{l+1}\right)$ and $\sum_{\lambda=l}^{r+1} x^{l}=c^{l}-y^{l}$. For $l=r$,

$$
x^{r}=g\left(E_{r}, c^{r}\right)=c^{r}-f\left(L_{r}, c^{r}\right)=c^{r}-y^{r}=c^{r}-y^{r}-\left(c^{r+1}-y^{r+1}\right)
$$

and $x^{r}=x^{r}+x^{r+1}=c^{r}-y^{r}$. Assume now that $x^{l+1}=c^{l}-y^{l}-\left(c^{l+1}-y^{l+1}\right)$ and $\sum_{\lambda=l+1}^{r+1} x^{l}=$ $c^{l+1}-y^{l+1}$ for some $l \in\{1, \ldots, r-1\}$. Then,

$$
\begin{aligned}
x^{l} & =g\left(E_{l}, c^{l}-\sum_{\lambda=l+1}^{r+1} x^{\lambda}\right) \\
& =g\left(E_{l}, c^{l}-\left(c^{l+1}-y^{l+1}\right)\right) \\
& =c^{l}-\left(c^{l+1}-y^{l+1}\right)-f\left(\sum_{\lambda=l}^{r} L_{\lambda}, c^{l}-\left(c^{l+1}-y^{l+1}\right)\right) \\
& =c^{l}-y^{l}-\left(c^{l+1}-y^{l+1}\right)
\end{aligned}
$$

where the third equality follows since $g$ is the dual rule of $f$ and $\sum_{i \in N} y_{i}^{l+1}=\sum_{\lambda=l+1}^{r} L_{\lambda}$, and the fourth equality is a direct consequence of the definition of $y^{l}$. Then,

$$
\begin{aligned}
\sum_{\lambda=l}^{r+1} x^{l} & =c^{l}-y^{l}-\left(c^{l+1}-y^{l+1}\right)+\sum_{\lambda=l+1}^{r+1} x^{l} \\
& =c^{l}-y^{l}-\left(c^{l+1}-y^{l+1}\right)+c^{l+1}-y^{l+1}=c^{l}-y^{l} .
\end{aligned}
$$

Therefore, for $l=1$,

$$
g^{\mathrm{Up}}(\underline{E}, c)=\sum_{\lambda=1}^{r+1} x^{\lambda}=c^{1}-y^{1}=c-f^{\mathrm{Up}}\left(\underline{F}^{1}, c-c^{2}+y^{2}\right) .
$$

Proof of Proposition 3.5. Let $(\underline{E}, c) \in \mathcal{B}^{N_{1}, \ldots, N_{r}}, k \in\{1, \ldots, r\}$, and $E_{k}^{\prime} \in \mathbb{R}_{+}$with $E_{k}^{\prime} \leq E_{k}$. Let $f^{\mathrm{Up}}(\underline{E}, c)=\sum_{l=1}^{r} x^{l}$ with $x^{l}, l=1, \ldots, r$ as in the description of the upwards mechanism. Let $\bar{x}^{k}=f\left(E_{k}-E_{k}^{\prime}, c^{k}-\sum_{\lambda=k+1}^{r} x^{\lambda}-x^{\prime k}\right)$ and $x^{\prime k}=f\left(E_{k}^{\prime}, c^{k}-\sum_{\lambda=k+1}^{r} x^{\lambda}\right)$. Since $f$ satisfies composition,

$$
x^{k}=f\left(E_{k}-E_{k}^{\prime}, c^{k}-\sum_{\lambda=k+1}^{r} x^{\lambda}-x^{\prime k}\right)+f\left(E_{k}^{\prime}, c^{k}-\sum_{\lambda=k+1}^{r} x^{\lambda}\right)=\bar{x}^{k}+x^{\prime k}
$$


and

$$
x^{\prime k}+\sum_{\lambda=k+1}^{r} x^{\lambda}=f\left(E_{k}^{\prime}, c^{k}-\sum_{\lambda=k+1}^{r} x^{\lambda}\right)+\sum_{\lambda=k+1}^{r} x^{\lambda}=f^{\mathrm{Up}}\left(\underline{E_{k}^{\prime}}+\underline{E}^{k+1}, c^{k}\right)=\tilde{x}^{k} .
$$

Besides, for $l \in\{1, \ldots, k-1\}$,

$$
x^{l}=f\left(E_{l}, c^{l}-\sum_{\lambda=l+1}^{r} x^{\lambda}\right)=f\left(E_{l}, c^{l}-\sum_{\lambda=l+1}^{k-1} x^{\lambda}-\tilde{x}^{k}-\bar{x}^{k}\right)
$$

and

$$
\sum_{\lambda=1}^{k-1} x^{\lambda}+\bar{x}^{k}=f^{\mathrm{Up}}\left(\underline{E}-\underline{E_{k}^{\prime}}-\underline{E}^{k+1}, c-\tilde{x}^{k}\right) .
$$

Then,

$$
\begin{aligned}
f^{\mathrm{Up}}(\underline{E}, c) & =\sum_{l=1}^{k-1} x^{l}+\bar{x}^{k}+x^{\prime k}+\sum_{\lambda=k+1}^{r} x^{\lambda} \\
& =f^{\mathrm{Up}}\left(\underline{E}-\underline{E_{k}^{\prime}}-\underline{E}^{k+1}, c-\tilde{x}^{k}\right)+f^{\mathrm{Up}}\left(\underline{E_{k}^{\prime}}+\underline{E}^{k+1}, c^{k}\right) .
\end{aligned}
$$

The following result is used in the proof of Proposition 4.1. It is a direct consequence of the definition of minimal rights and the proof is, therefore, omitted. For (ii), we refer to $\mathrm{O}^{\prime} \mathrm{Neill}$ (1982), Curiel et al. (1987).

Lemma A.1. Let $\left(\underline{E_{k}}, c\right) \in \mathcal{B}^{N_{1}, \ldots, N_{r}}$.

(i) $m_{N_{l}}=\mathbf{0}_{N_{l}}, \overline{l \in}\{1, \ldots, k-1\}$.

(ii) $\sum_{\lambda \in I} m\left(N_{\lambda}\right) \leq\left(E_{k}-\sum_{\lambda \in\{k \ldots, r\} \backslash I} c\left(N_{\lambda}\right)\right)_{+}, I \subseteq\{k, \ldots, r\}$.

\section{Proof of Proposition 4.1.}

(i) Let $f$ be a bankruptcy rule satisfying invariance under claims truncation. Let $(\underline{E}, c) \in$ $\mathcal{B}^{N_{1}, \ldots, N_{r}}$. We first show that $f^{\mathrm{D}}$ satisfies invariance under claims truncation. Let $L_{\lambda}^{\underline{E}}=L_{\lambda}\left(\underline{E}, c^{\underline{E}}\right)=$ $c^{\underline{E}}\left(N_{\lambda}\right)-E_{\lambda}$. By definition of $c^{\underline{E}}, L_{\lambda}^{\underline{E}} \leq L_{\lambda}$ for each $\lambda \in\{1, \ldots, r\}$. Besides, let $f^{\mathrm{D}}\left(\underline{E}, c^{\underline{E}}\right)=$ $\left(x_{N_{1}}^{1}, \ldots, x_{N_{r}}^{r}\right)$ with $x^{1}, \ldots, x^{r}$ as in the description of the downstream mechanism and let $x_{i_{1}}^{0}=$ 0 . For $l \in\{1, \ldots, r-1\}, x_{i_{l+1}}^{l} \leq E_{l}+x_{i_{l}}^{l-1} \leq \sum_{\mu=1}^{l} E_{\mu}$. Moreover,

$$
\begin{gathered}
\min \left\{E_{l}+x_{i_{l}}^{l-1}, \sum_{\mu=l+1}^{r} L \frac{E}{\mu}\right\}=E_{l}+x_{i_{l}}^{l-1} \\
\Rightarrow \min \left\{E_{l}+x_{i_{l}}^{l-1}, \sum_{\mu=l+1}^{r} L_{\mu}\right\}=E_{l}+x_{i_{l}}^{l-1}, \\
\min \left\{E_{l}+x_{i_{l}}^{l-1}, \sum_{\mu=l+1}^{r} L \frac{E}{\mu}\right\}<E_{l}+x_{i_{l}}^{l-1} \\
\Rightarrow c_{N_{l+1}}^{E}=c_{N_{l+1}}, \ldots, c_{N_{r}}^{E}=c_{N_{r}} .
\end{gathered}
$$

Equation (4a) follows directly from $L_{\mu}^{E} \leq L_{\mu}$ for each $\mu \in\{1, \ldots, r\}$. To show Equation (4b), 
we proceed by contradiction. Suppose that there exist $\lambda \in\{l+1, \ldots, r\}$ and $i \in N_{\lambda}$ with $c_{i}>$ $\sum_{\mu=1}^{\lambda} E_{\mu}$. Then, $c_{i}^{E}=\sum_{\mu=1}^{\lambda} E_{\mu}$, the definition of $L_{\mu}^{\frac{E}{\mu}}$, and the assumption on sequencing bankruptcy problems imply

$$
\begin{aligned}
E_{l}+x_{i_{l}}^{l-1} & >\sum_{\mu=l+1}^{r} L_{\underline{\mu}}^{\underline{E}}=\sum_{\mu=l+1}^{\lambda-1}\left(c^{\underline{E}}\left(N_{\mu}\right)-E_{\mu}\right)+\sum_{\mu=1}^{\lambda-1} E_{\mu}+c^{\underline{E}}\left(N_{\lambda} \backslash\{i\}\right)+\sum_{\mu=\lambda+1}^{r} L_{\mu}^{\underline{E}} \\
& \geq \sum_{\mu=l+1}^{\lambda-1}\left(c^{\underline{E}}\left(N_{\mu}\right)-E_{\mu}\right)+\sum_{\mu=1}^{\lambda-1} E_{\mu}+c^{\underline{E}}\left(N_{\lambda} \backslash\{i\}\right) \geq \sum_{\mu=1}^{l} E_{\mu} \geq E_{l}+x_{i_{l}}^{l-1}
\end{aligned}
$$

which establishes a contradiction.

Then, $c_{N_{1}}^{E_{1}} \in \mathbb{R}^{N_{1}}$ defined by $c_{i}^{E_{1}}=\min \left\{c_{i}, E_{1}\right\}=\min \left\{c_{i}^{E}, E_{1}\right\}$ for all $i \in N_{1}$ implies

$$
\begin{aligned}
x^{1} & =f\left(E_{1},\left(c_{N_{1}}^{E}, \sum_{\lambda=2}^{r} L \frac{E}{\lambda}\right)\right)=f\left(E_{1},\left(c_{N_{1}}^{E_{1}}, \min \left\{E_{1}, \sum_{\lambda=2}^{r} L \frac{E}{\lambda}\right\}\right)\right) \\
& =f\left(E_{1},\left(c_{N_{1}}, \sum_{\lambda=2}^{r} L_{\lambda}\right)\right)
\end{aligned}
$$

where the second equality follows by invariance under claims truncation, and the last equality is a direct consequence of invariance under claims truncation and Equations (4a) and (4b). Then,

$$
f_{N_{1}}^{\mathrm{D}}\left(\underline{E}, c^{\underline{E}}\right)=x_{N_{1}}^{1}=f_{N_{1}}^{\mathrm{D}}(\underline{E}, c)
$$

Following the same reasoning as above, we get $f^{\mathrm{D}}\left(\underline{E}, c^{\underline{E}}\right)=f^{\mathrm{D}}(\underline{E}, c)$.

Next, we show that $f^{2 s}$ satisfies invariance under claims truncation. Let

$$
d_{l}^{E}=\min \left\{c^{\underline{E}}\left(N_{l}\right), \sum_{\lambda=1}^{l} E_{\lambda}\right\}=\min \left\{c\left(N_{l}\right), \sum_{\lambda=1}^{l} E_{\lambda}\right\}=d_{l}
$$

for $l \in\{1, \ldots, r\}$ and

$$
x^{\underline{E}}=f\left(\sum_{\lambda=1}^{r} E_{\lambda},\left(d_{1}^{E}, \ldots, d \frac{E}{r}\right)\right)=f\left(\sum_{\lambda=1}^{r} E_{\lambda},\left(d_{1}, \ldots, d_{r}\right)\right)=x .
$$

Then, $x=x^{\underline{E}} \leq d^{E} \leq d$ and invariance under claims truncation imply

$$
f\left(x_{l}, c_{N_{l}}^{\underline{E}}\right)=f\left(x_{l}, c_{N_{l}}\right) \text { for } l \in\{1, \ldots, r\}
$$

and, therefore, $f^{2 s}\left(\underline{E}, c^{\underline{E}}\right)=f^{2 s}(\underline{E}, c)$.

(ii) Let $f$ be a bankruptcy rule satisfying invariance under claims truncation. By (i), $f^{\mathrm{D}}$ and $f^{2 s}$ satisfy invariance under claims truncation. As a consequence, $f^{\mathrm{D}}$ and $f^{2 \mathrm{~s}}$ satisfy weak 
invariance under claims truncation.

(iii) Let $f$ be a bankruptcy rule satisfying minimal rights first. Let $\left(\underline{E_{k}}, c\right) \in \mathcal{B}^{N_{1}, \ldots, N_{r}}$. For $l \in\{1, \ldots, r\}$, let $M_{l}=m\left(N_{l}\right)$ and $M=\sum_{l=1}^{r} M_{l}$. We first show that $f^{\mathrm{D}}$ satisfies weak minimal rights first. For $l \in\{1, \ldots, r-1\}$, let $\bar{N}_{l}=N_{l} \cup\left\{i_{l+1}\right\}$. By Lemma A.1 (i), $M=\sum_{l=k}^{r} M_{l}$.

For $l \in\{1, \ldots, k-1\}, f_{N_{l}}^{\mathrm{D}}\left(\underline{E_{k}}, c\right)=f_{N_{l}}^{\mathrm{D}}\left(\underline{E_{k}-M}, c-m\right)=\mathbf{0}_{N_{l}}=m_{N_{l}}$ implies the result for $N_{1} \cup \ldots \cup N_{k-1}$. Next, we show the result for $l=k$. Let

$$
m^{k}=m\left(E_{k},\left(c_{N_{k}}, \sum_{\lambda=k+1}^{r} c\left(N_{\lambda}\right)\right)\right)
$$

and

$$
m^{k, M}=m\left(E_{k}-M,\left(c_{N_{k}}-m_{N_{k^{\prime}}} \sum_{\lambda=k+1}^{r}\left(c\left(N_{\lambda}\right)-M_{\lambda}\right)\right)\right) .
$$

Besides, let $M^{k}=m^{k}\left(N_{k} \cup\left\{i_{k+1}\right\}\right)$ and $M^{k, M}=m^{k, M}\left(N_{k} \cup\left\{i_{k+1}\right\}\right)$. Then, by definition of minimal right and Lemma A.1 (ii), $m_{i}^{k}=m_{i}^{k, M}+m_{i}$ for $i \in N_{k}$ and $m_{i_{k+1}}^{k}=m_{i_{k+1}}^{k, M}+\sum_{\lambda=k+1}^{r} M_{\lambda}$. Then, $M^{k}=M+M^{k, M}$ and

$$
\begin{aligned}
f\left(E_{k},\left(c_{N_{k^{\prime}}}, \sum_{\lambda=k+1}^{r} c\left(N_{\lambda}\right)\right)\right)=m^{k}+f\left(E_{k}-M^{k},\left(c_{N_{k^{\prime}}} \sum_{\lambda=k+1}^{r} c\left(N_{\lambda}\right)\right)-m^{k}\right) \\
=\left(m_{N_{k^{\prime}}}, \sum_{\lambda=k+1}^{r} M_{\lambda}\right)+m^{k, M} \\
\quad+f\left(E_{k}-M-M^{k, M},\left(c_{N_{k}}-m_{N_{k^{\prime}}} \sum_{\lambda=k+1}^{r}\left(c\left(N_{\lambda}\right)-M_{\lambda}\right)\right)-m^{k, M}\right) \\
=\left(m_{N_{k^{\prime}}} \sum_{\lambda=k+1}^{r} M_{\lambda}\right)+f\left(E_{k}-M,\left(c_{N_{k}}-m_{N_{k^{\prime}}} \sum_{\lambda=k+1}^{r}\left(c\left(N_{\lambda}\right)-M_{\lambda}\right)\right)\right)
\end{aligned}
$$

where the first and third equalities follow by minimal rights first. Then,

$$
f_{N_{k}}^{\mathrm{D}}\left(\underline{E_{k}}, c\right)=m_{N_{k}}+f_{N_{k}}^{\mathrm{D}}\left(\underline{E_{k}-M}, c-m\right) \text { and } x_{i_{k+1}}^{k}=x_{i_{k+1}^{k, M}}^{k,}+\sum_{\lambda=k+1}^{r} M_{\lambda}
$$

with $\left(E_{k}-c\left(N_{k}\right)\right)_{+} \leq x_{i_{k+1}}^{k} \leq \sum_{\lambda=k+1}^{r} c\left(N_{\lambda}\right)$.

Next, we show the result for $k+1$. Let $m^{k+1}=m\left(x_{i_{k+1}}^{k}\left(c_{N_{k+1}}, \sum_{\lambda=k+2}^{r} c\left(N_{\lambda}\right)\right)\right)$ and $m^{k+1, M}=$ $m\left(x_{i_{k+1}^{k, M}},\left(c_{N_{k+1}}-m_{N_{k+1}}, \sum_{\lambda=k+2}^{r}\left(c\left(N_{\lambda}\right)-M_{\lambda}\right)\right)\right)$. Besides, let $M^{k+1}=m_{i}^{k+1}\left(N_{k+1} \cup\left\{i_{k+2}\right\}\right)$ and $M^{k+1, M}=m_{i}^{k+1, M}\left(N_{k+1} \cup\left\{i_{k+2}\right\}\right)$. Using $x_{i_{k+1}}^{k}=x_{i_{k+1}}^{k, M}+\sum_{\lambda=k+1}^{r} M_{\lambda}$, we have

$$
m_{i}^{k+1}=\left(c_{i}-\left(\sum_{\lambda=k+1}^{r} c\left(N_{\lambda}\right)-x_{i_{k+1}}^{k}\right)\right)_{+} \text {for } i \in N_{k+1},
$$




$$
\begin{aligned}
m_{i_{k+2}}^{k+1} & =\left(x_{i_{k+1}}^{k}-c\left(N_{k+1}\right)\right)_{+} \\
m_{i}^{k+1, M} & =\left(c_{i}-m_{i}-\left(\sum_{\lambda=k+1}^{r} c\left(N_{\lambda}\right)-x_{i_{k+1}}^{k}\right)\right)_{+} \text {for } i \in N_{k+1}, \\
m_{i_{k+2}}^{k+1, M} & =\left(x_{i_{k+1}}^{k}-c\left(N_{k+1}\right)-\sum_{\lambda=k+2}^{r} M_{\lambda}\right)_{+}
\end{aligned}
$$

For $i \in N_{k+1}$,

$$
m_{i}^{k+1, M}+m_{i}=\max \left\{m_{i}, c_{i}-\left(\sum_{\lambda=k+1}^{r} c\left(N_{\lambda}\right)-x_{i_{k+1}}^{k}\right)\right\}=m_{i}^{k+1}
$$

where the second equality is a direct consequence of $x_{i_{k+1}}^{k} \geq\left(E_{k}-c\left(N_{k}\right)\right)_{+} \geq E_{k}-c\left(N_{k}\right)$ and the definition of $m_{i}$. Moreover,

$$
\begin{aligned}
m_{i_{k+2}}^{k+1, M}+\sum_{\lambda=k+2}^{r} M_{\lambda} & =\max \left\{\sum_{\lambda=k+2}^{r} M_{\lambda}, x_{i_{k+1}}^{k}-c\left(N_{k+1}\right)\right\} \\
& =\max \left\{0, x_{i_{k+1}}^{k}-c\left(N_{k+1}\right)\right\}=m_{i_{k+2}}^{k+1}
\end{aligned}
$$

where the second equality follows since $x_{i_{k+1}}^{k} \geq\left(E_{k}-c\left(N_{k}\right)\right)_{+} \geq E_{k}-c\left(N_{k}\right)$ and Lemma A.1 (ii). Then, $\sum_{\lambda=k+1}^{r} M_{\lambda}+M^{k+1, M}=M^{k+1}$. Therefore,

$$
\begin{aligned}
f\left(x_{i_{k+1}}^{k},\left(c_{N_{k+1}}, \sum_{\lambda=k+2}^{r} c\left(N_{\lambda}\right)\right)\right) \\
=m^{k+1}+f\left(x_{i_{k+1}}^{k}-M^{k+1},\left(c_{N_{k+1}}, \sum_{\lambda=k+2}^{r} c\left(N_{\lambda}\right)\right)-m^{k+1}\right) \\
=\left(m_{N_{k+1}}, \sum_{\lambda=k+2}^{r} M_{\lambda}\right)+m^{k+1, M} \\
\quad+f\left(x_{i_{k+1}^{k}}^{k}-\sum_{\lambda=k+1}^{r} M_{\lambda}-M^{k+1, M},\left(c_{N_{k+1}}-m_{N_{k+1}}, \sum_{\lambda=k+2}^{r}\left(c\left(N_{\lambda}\right)-M_{\lambda}\right)\right)-m^{k+1, M}\right) \\
=\left(m_{N_{k+1}}, \sum_{\lambda=k+2}^{r} M_{\lambda}\right)+f_{N_{k+1}}\left(x_{i_{k+1}^{k}}^{k}-\sum_{\lambda=k+1}^{r} M_{\lambda},\left(c_{N_{k+1}}-m_{N_{k+1}}, \sum_{\lambda=k+2}^{r}\left(c\left(N_{\lambda}\right)-M_{\lambda}\right)\right)\right)
\end{aligned}
$$

where the first and third equalities follow by minimal rights first. Then,

$$
f_{N_{k+1}}^{\mathrm{D}}\left(\underline{E_{k}}, c\right)=m_{N_{k+1}}+f_{N_{k+1}}^{\mathrm{D}}\left(\underline{E_{k}-M}, c-m\right) \text { and } x_{i_{k+2}}^{k+1}=x_{i_{k+2}}^{k+1, M}+\sum_{\lambda=k+2}^{r} M_{\lambda}
$$

with $\left(E_{k}-\sum_{\lambda=k}^{k+1} c\left(N_{\lambda}\right)_{+} \leq x_{i_{k+1}}^{k} \leq \sum_{\lambda=k+2}^{r} c\left(N_{\lambda}\right)\right.$.

Following the same reasoning for $k+2, \ldots, r$, it can be seen that $f^{\mathrm{D}}$ satisfies weak minimal rights first. 
Next, we show that $f^{2 s}$ satisfies weak minimal rights first. Let $d, d^{M} \in \mathbb{R}^{\{1, \ldots, r\}}$ where $d$ and $d^{M}$ are defined as in the description of the two-steps mechanism for $\left(\underline{E_{k}}, c\right)$ and $\left(\underline{E_{k}-M}, c-m\right)$, respectively. First,

$$
d^{M}=d-\left(M_{1}, \ldots, M_{r}\right)
$$

To see this, let $l \in\{1, \ldots, k-1\}$, then, $d_{l}^{M}=d_{l}=M_{l}=0$ and $d_{l}^{M}+M_{l}=0=d_{l}$. Let $l \in\{k, \ldots, r\}$. If $c\left(N_{l}\right) \geq E_{k}$, then, $M_{\lambda}=0$ for all $\lambda \neq l$, which implies $M=M_{l}$ and

$$
d_{l}^{M}+M_{l}=\min \left\{E_{k}-M, c\left(N_{l}\right)-M_{l}\right\}+M_{l}=\min \left\{E_{k}, c\left(N_{l}\right)\right\}=d_{l} .
$$

If $c\left(N_{l}\right)<E_{k}$, then, $M_{\lambda}=0$ for $\lambda \in\{1, \ldots, k-1\}$ and Lemma A.1 (ii) imply $M-M_{l} \leq$ $\left(E_{k}-c\left(N_{l}\right)\right)_{+}=E_{k}-c\left(N_{l}\right)$ and

$$
d_{l}^{M}+M_{l}=\min \left\{E_{k}-M+M_{l}, c\left(N_{l}\right)\right\}=c\left(N_{l}\right)=d_{l} .
$$

Second,

$$
\left(M_{1}, \ldots, M_{r}\right)+m\left(E_{k}-M, d^{M}\right)=m\left(E_{k}, d\right)
$$

To see this, by Equation (5) and definition of $m_{l}\left(E_{k}-M, d^{M}\right)$,

$$
M_{l}+m_{l}\left(E_{k}-M, d^{M}\right)=\max \left\{M_{l}, d_{l}-\left(\sum_{\lambda=1}^{r} d_{\lambda}-E_{k}\right)\right\}
$$

Let $l \in\{1, \ldots, k-1\}$. Then, $M_{l}=d_{l}=0$ implies $M_{l}+m_{l}\left(E_{k}-M, d^{M}\right)=0=m_{l}\left(E_{k}, d\right)$. Let $l \in\{k, \ldots, r\}$. If $c\left(N_{\lambda}\right) \leq E_{k}$ for all $\lambda \in\{k, \ldots, r\}$, then, $d_{\lambda}=c\left(N_{\lambda}\right)$ for all $\lambda \in\{k, \ldots, r\}$ and

$$
\begin{aligned}
M_{l}+m_{l}\left(E_{k}-M, d^{M}\right) & =\max \left\{M_{l}, d_{l}-\left(\sum_{\lambda=1}^{r} d_{\lambda}-E_{k}\right)\right\} \\
& =\max \left\{0, d_{l}-\left(\sum_{\lambda=1}^{r} d_{\lambda}-E_{k}\right)\right\}=m_{l}\left(E_{k}, d\right)
\end{aligned}
$$

where the second equality is a direct consequence of $d_{\lambda}=c\left(N_{\lambda}\right)$ for all $\lambda \in\{k, \ldots, r\}$ and $M_{l} \leq\left(E_{k}-\left(\sum_{\substack{\lambda=k \\ \lambda \neq l}}^{r} c\left(N_{\lambda}\right)\right)\right)_{+}$by Lemma A.1 (ii). If $c\left(N_{\bar{l}}\right)>E_{k}$ for some $\bar{l} \in\{k, \ldots, r\}$, then, $d_{\bar{l}}=E_{k}<c\left(N_{\bar{l}}\right), M_{\lambda}=0$ for all $\lambda \in\{1, \ldots, r\} \backslash\{\bar{l}\}$ and $M=M_{\bar{l}}$. Then, for $l \in\{k, \ldots, r\} \backslash\{\bar{l}\}$,

$$
M_{l}+m_{l}\left(E_{k}-M, d^{M}\right)=\max \left\{0, d_{l}-\left(\sum_{\lambda=1}^{r} d_{\lambda}-E_{k}\right)\right\}=m_{l}\left(E_{k}, d\right) .
$$

For $l=\bar{l}$, if $M_{\bar{l}}=0$,

$$
M_{\bar{l}}+m_{\bar{l}}\left(E_{k}-M, d^{M}\right)=\max \left\{0, d_{\bar{l}}-\left(\sum_{\lambda=1}^{r} d_{\lambda}-E_{k}\right)\right\}=m_{\bar{l}}\left(E_{k}, d\right)
$$


if $M_{\bar{l}}>0$, then, by Lemma A.1 (ii), $E_{k}>\sum_{\substack{\lambda=k \\ \lambda \neq l}}^{r} c\left(N_{\lambda}\right)$, which implies $d_{l}=c\left(N_{l}\right)$ for all $l \in$ $\{k, \ldots, r\} \backslash\{\bar{l}\} ;$ then,

$$
\begin{aligned}
M_{\bar{l}}+m_{\bar{l}}\left(E_{k}-M, d^{M}\right) & =\max \left\{M_{\bar{l}}, d_{\bar{l}}-\left(\sum_{\lambda=1}^{r} d_{\lambda}-E_{k}\right)\right\} \\
& =\max \left\{M_{\bar{l}}, E_{k}-\sum_{\substack{\lambda=k \\
\lambda \neq \bar{l}}}^{r} c\left(N_{\lambda}\right)\right\} \\
& =\max \left\{0, E_{k}-\sum_{\substack{\lambda=k \\
\lambda \neq \bar{l}}}^{r} c\left(N_{\lambda}\right)\right\}=m_{\bar{l}}\left(E_{k}, d\right)
\end{aligned}
$$

where the third equality is a direct consequence of Lemma A.1 (ii).

Let $x=f\left(E_{k}, d\right)$ and $y=f\left(E_{k}-M, d^{M}\right)$. Then,

$$
\begin{aligned}
x= & m\left(E_{k}, d\right)+f\left(E_{k}-\sum_{\lambda=1}^{r} m_{\lambda}\left(E_{k}, d\right), d-m\left(E_{k}, d\right)\right) \\
= & \left(M_{1}, \ldots, M_{r}\right)+m\left(E_{k}-M, d^{M}\right) \\
& +f\left(E_{k}-M-\sum_{\lambda=1}^{r} m_{\lambda}\left(E_{k}-M, d^{M}\right), d-\left(M_{1}, \ldots, M_{r}\right)-m\left(E_{k}-M, d^{M}\right)\right) \\
= & \left(M_{1}, \ldots, M_{r}\right)+y
\end{aligned}
$$

where the first equality follows by minimal rights first, the second equality is a direct consequence of Equation (6), and the third equality follows by minimal rights first and Equation (5).

Next, we show

$$
m_{N_{l}}+m\left(x_{l}-M_{l}, c_{N_{l}}-m_{N_{l}}\right)=m\left(x_{l}, c_{N_{l}}\right) \text { for all } l \in\{k, \ldots, r\} .
$$

Let $i \in N_{l}$, then,

$$
\begin{aligned}
m_{i}+m_{i}\left(x_{l}-M_{l}, c_{N_{l}}-m_{N_{l}}\right) & =\max \left\{m_{i}, c_{i}-\left(c\left(N_{l}\right)-x_{l}\right)\right\} \\
& =\max \left\{0, c_{i}-\left(c\left(N_{l}\right)-x_{l}\right)\right\}=m_{i}\left(x_{l}, c_{N_{l}}\right)
\end{aligned}
$$

where the second equality is a direct consequence of $x_{l} \geq\left(E_{k}-\sum_{\substack{\lambda=k \\ \lambda \neq l}}^{r} c\left(N_{l}\right)\right)_{+} \geq E_{k}-\sum_{\substack{\lambda=k \\ \lambda \neq l}}^{r} c\left(N_{l}\right)$ and the definition of $m_{i}$.

For $l \in\{1, \ldots, k-1\}$,

$$
m_{N_{l}}+f_{N_{l}}^{2 \mathrm{~s}}\left(\underline{E_{k}-M}, c-m\right)=\mathbf{0}_{N_{l}}=f_{N_{l}}^{2 \mathrm{~s}}\left(\underline{E_{k}}, c\right)
$$


and, for $l \in\{k, \ldots, r\}$,

$$
\begin{aligned}
m_{N_{l}}+ & f_{N_{l}}^{2 s}\left(\underline{E_{k}-M}, c-m\right)=m_{N_{l}}+f\left(y_{l}, c_{N_{l}}-m_{N_{l}}\right) \\
= & m_{N_{l}}+f\left(x_{l}-M_{l}, c_{N_{l}}-m_{N_{l}}\right) \\
= & m_{N_{l}}+m\left(x_{l}-M_{l}, c_{N_{l}}-m_{N_{l}}\right) \\
& +f\left(x_{l}-M_{l}-\sum_{i \in N_{l}} m_{i}\left(x_{l}-M_{l}, c_{N_{l}}-m_{N_{l}}\right),\right. \\
& \left.c_{N_{l}}-m_{N_{l}}-m\left(x_{l}-M_{l}, c_{N_{l}}-m_{N_{l}}\right)\right) \\
= & m\left(x_{l}, c_{N_{l}}\right)+f\left(x_{l}-\sum_{i \in N_{l}} m_{i}\left(x_{l}, c_{N_{l}}\right), c_{N_{l}}-m\left(x_{l}, c_{N_{l}}\right)\right) \\
= & f\left(x_{l}, c_{N_{l}}\right)=f_{N_{l}}^{2 s}\left(\underline{E_{k}}, c\right)
\end{aligned}
$$

where the first and sixth equalities are direct consequence of the definition of $f^{2 s}$, the second equality follows because $x=\left(M_{1}, \ldots, M_{r}\right)+y$, the third and fifth equalities follow by minimal rights first, and the fourth equality is a direct consequence of Equation (7).

Acknowledgments. The authors would like to thank Peter Borm for his suggestions and comments for the improvement of this paper.

Usual caveat applies. Financial support from Universitat Rovira i Virgili and Generalitat de Catalunya (2017PFRURV-B2-53 and 2017 SGR 770) and Ministerio de Economía y Competitividad (ECO2016-75410-P (AEI/FEDER, UE) is acknowledged.

\section{References}

Ambec, S. and Sprumont, Y. (2002), 'Sharing a river', Journal of Economic Theory 107, 453-462.

Ansink, E. and Weikard, H.-P. (2012), 'Sequential sharing rules for river sharing problems', Social Choice and Welfare 38, 187-210.

Aumann, R. and Maschler, M. (1985), 'Game theoretic analysis of a bankruptcy problem from the talmud', Journal of Economic Theory 36, 195-213.

Bergantiños, G. and Moreno-Ternero, J. D. (2015), 'The axiomatic approach to the problem of sharing the revenue from museum passes', Games and Economic Behavior 89, 78-92.

Bergantiños, G. and Moreno-Ternero, J. D. (2016), 'A new rule for the problem of sharing the revenue from museum passes', Operations Research Letters 44, 208-211.

Borm, P., Carpente, L., Casas-Méndez, B. and Hendrickx, R. (2005), ‘The constrained equal awards rule for bankruptcy problems with a priori unions', Annals of Operations Research 137, 211-227.

Casas-Mendez, B., Fragnelli, V. and Garcia-Jurado, I. (2011), 'Weighted bankruptcy rules and the museum pass problem', European Journal of Operational Research 215, 161-168.

Casas-Mendez, B., Fragnelli, V. and Garcia-Jurado, I. (2014), 'A survey of allocation rules for the museum pass problem', Journal of Cultural Economics 38, 191-205. 
Curiel, I. J., Maschler, M. and Tijs, S. (1987), ‘Bankruptcy games', Zeitschrift für Operations Research 31, A 143-A 159.

Dagan, N. (1996), 'New characterizations of old bankruptcy rules', Social Choice and Welare 13, 51-59.

Duro Moreno, J. A., Giménez Gómez, J. M. and Vilella, C. (2018), ‘The allocation of $\mathrm{CO}_{2}$ emissions as a claims problem', Centre de Recerca en Economia Industrial i Economia Pública . Universitat Rovira i Virgili, Spain.

Estévez-Fernández, A. (2012), 'A game theoretical approach to sharing penalties and rewards in projects', European Journal of Operational Research 216, 647-657.

Estévez-Fernández, A., Borm, P. and Hamers, H. (2007), 'Project games', International Journal of Game Theory 36, 149176.

Estévez-Fernández, A., Borm, P. and Hamers, H. (2012), 'A note on passepartout problems', International Game Theory Review 14, 1-9.

Estévez-Fernández, A., Borm, P. and Lazarova, E. (2016), 'Transfers and exchange-stability in two-sided matching problems', Theory and Decision 81, 53-71.

Herrero, C. and Villar, A. (2001), 'The three musketeers: four classical solutions to bankruptcy problems', Mathematical Social Sciences 42, 307-328.

Moulin, H. (1987), 'Equal or proportional division of a surplus, and other methods', International Journal of Game Theory 16, 161-186.

Moulin, H. (2000), 'Priority rules and other asymmetric rationing methods', Econometrica 68, 643-684.

O’Neill, B. (1982), 'A problem of rights arbitration from the Talmud', Mathematical Social Sciences 2(4), 345-371.

Thomson, W. (2003), 'Axiomatic and game-theoretic analysis of bankruptcy and taxation problems: a survey', Mathematical Social Sciences 45, 249-297.

Thomson, W. (2015), 'Axiomatic and game-theoretic analysis of bankruptcy and taxation problems: an update', Mathematical Social Sciences 74, 867-880.

Young, H. P. (1988), 'Distributive justice in taxation', Journal of Economic Theory 44, 321-335. 